\title{
VARIATIONAL MICRO-MACRO MODEL AND DEFORMATION TEXTURES PREDICTED FOR STEELS ${ }^{1}$
}

\author{
MAYEUL ARMINJON and DIDIER IMBAULT \\ Laboratoire "Sols, Solides, Structures" [Université J. Fourier / Inst. Nat. \\ Polytechnique de Grenoble / C.N.R.S], B.P. 53 X, 38041 Grenoble cedex, France
}

(Received 20 November 1995)

\begin{abstract}
This variational micro-macro model is presented in the context of the "pure" and "relaxed" Taylor models, from which it evolved, but also in relation to the Sachs-type models and, more generally, in the context of volume-fraction or "one-point" models. It will indeed be shown that any one-point model must set statistical assumptions and that, to this general frame, the present model adds only one essential assumption- namely a plausible principle of minimal inhomogeneity. The numerical implementation is summarized. Predictions for the strain distribution are discussed. Predicted deformation textures are compared with measurements on steel sheets.
\end{abstract}

KEY WORDS: Polycrystal model, statistical homogeneity, variational principle, strain inhomogeneity, deformation texture, steel.

\section{INTRODUCTION}

Prediction of deformation textures is one of the primary goals for models that aim at making a micro-macro transition in polycrystals. Firstly, because they are so important in connection with the anisotropy of polycrystalline metals and rocks. Secondly, because they provide a sensitive test of such models. The latter reason may be worth to be developed somewhat. As opposed to purely phenomenological models, whose main interest may be to concentrate empirical information in a suitable form, a micro-macro model should bring some new information on the macro-scale from micro-scale information, and vice-versa. But in the micro-to-macro transition, we are sometimes in the following situation: the number $P$ of $a$ priori unknown parameters that are needed to run the model, is nearly equal to the total number $M$ of macroscopic data which are used, in a first step, to calibrate the model (thus to give values to the unknown parameters) and, in a second step, to test the model (thus to "predict" values for the macroscopic data, yet using the now known parameters). In a such situation, this test can obviously not be very convincing.

For instance, the critical shear stresses $\tau_{k}^{c}$, in a model for polycrystal plasticity based on Schmid's law, are essentially not accessible by direct measurement. If one wants to predict macroscopic stresses, it is hence nearly unavoidable to "adjust" the values according to macroscopic mechanical measurements. Depending on the scheme adopted

\footnotetext{
${ }^{1}$ This paper is dedicated to the memory of Hsun HU.
} 
for strain-hardening in the constituent crystals, the knowledge of the $\tau_{k}^{c}$ values in the model will be determined by some parameters, say $a_{1}, \ldots, a_{p}$, thus $P \geq p$. Hence, at least the number $p$ will have to be subtracted from $M$ to evaluate the number of data remaining for the true test of the model. Many models need not only the critical shear stresses but other "physical", yet not directly measurable parameters, say $b_{1}, \ldots ., b_{q}$, thus $P=p+q$. Examples of such $b_{j}$ parameters are the "plastic accommodation parameter" $\alpha$ of the simplified self-consistent scheme proposed by Berveiller \& Zaoui (1979), or the "average inhomogeneity of the actual strain-rate distribution)" $r_{\mathrm{o}}$ of the here-discussed model (Arminjon, 1991a; Arminjon et al., 1994). The more complex model one builds, the larger will be the number $P$ of $a$ priori unknown parameters it involves. However, some important macroscopic quantities may often be predicted with relatively few unknown (adjusted) parameters. Here, "often" means: in several rather simple models, among which some widely used ones. An example is provided by the Lankford coefficients $R$. When the critical shear stress is assumed uniform in a polycrystal (case of cubic symmetry with all-equivalent glide systems and "statistically uniform" hardening), the $R$ values may be computed as soon as one knows the $b_{j}$ parameters and the texture function (the ODF), the latter being measurable.

In contrast, the macro-to-micro transition is inherently a risky affair, hence an interesting one. The prediction of deformation textures is really a good example. Under the same assumption or uniform $\tau_{k}^{c}$ 's, this prediction also depends on the $b_{j}$ parameters only- and, of course, of the macroscopic deformation history and the possible initial texture: these are measurable input data for the model. But the testable output parameters are now the values of the "deformed" ODF with a reasonable angular resolution $\left(5^{\circ}\right.$, say) or, what is equivalent but often much more compact, the texture coefficients (Bunge, 1969). Thus, for each deformation mode at each deformation step, we have here several hundreds of output parameters. If we think of the approximations that one must do to get a model depending on one parameter only (as the model used here, or the Berveiller-Zaoui model), we may say that it would be quite surprising if such model were able to predict these deformation textures within good experimental accuracy. In our opinion, there is no doubt that a model without any variable parameter, such as e.g. the pure Taylor model, can absolutely not be expected to do this.

In this paper, we present a model which, indeed, does not predict deformation textures within experimental accuracy, but which seems to reasonably approach this goal. First, the new model will be presented in the context of the Taylor model and the "relaxed" Taylor model (Mecking, 1980; Kocks and Canova, 1981; Van Houtte, 1981), since the new model started as an extension of the former ones; in this course, some other models, e.g. the Sachs model and the modified Sachs model (Leffers, 1968; Pedersen and Leffers, 1987), will have to be referred to. Rather than on formal arguments, it will be insisted on the physical background, which is a bit more subtle a point than one might think, if one would look merely at the simplicity of the basic algorithm. Full mathematical justifications, depending on quite general statistical assumptions, have been provided by Arminjon (1991 a-b) for some crucial properties used in the model. It will be distinguished between those properties and one physical assumption (Arminjon et al., 1994) which cannot be derived from the basic laws of deterministic mechanics. The main features of the recently completed numerical implementation will be presented. Some salient aspects of the inhomogeneous distributions of strain-rate and stress which are numerically predicted by the model, will be discussed. Lastly, the deformation textures newly predicted for a bcc polycrystal deforming by $<111>$-pencil-glide will be compared with experimental data for low-carbon steel sheets, already used by Arminjon and Donadille (1990). 


\section{PRESENTATION OF THIS MODEL IN THE CONTEXT OF OTHER MODELS}

Taylor's model and the problem of strain compatibility in models with non-uniform strain

Voigt (1887) proposed to calculate the macroscopic elastic moduli of a polycrystal by averaging the microscopic ones, and recognized that this amounts to assuming that the elastic strain is uniform in the elastically deformed polycrystal; much later, Hill (1952) proved that Voigt's model leads, for a statistically isotropic polycrystal, to an overestimate of the bulk and rigidity moduli. Taylor (1938) proposed to use just the same assumption of a uniform strain (increment) to calculate the yield locus of a plastically deformed polycrystal from the yield locus of the crystal, and it did not take that long time until Bishop and Hill (1951) proved that Taylor's model leads, and also for a textured polycrystal, to an overestimate (an approach from outside) of the yield locus. (Thus it seems that we have here a case where elasticity is not easier than plasticity.) In these theoretical proofs by Bishop and Hill (1951) and by Hill (1952), a crucial assumption is the no-correlation condition between actual microscopic fields of stress and strain (or strain-rate, which is more relevant to plasticity), thus for the stress $\sigma^{\prime}$ and the strain-rate $\mathbf{d}$ :

$$
\overline{\sigma^{\prime}: \mathbf{d}}=\overline{\sigma^{\prime}}: \overline{\mathbf{d}}
$$

In Eq. (1) the double point means scalar product of tensors, thus $\sigma^{\prime}: \mathbf{d}=\sigma_{i j}^{\prime} d_{i j}$, and the bar denotes volume average over a representative volume element (RVE). The prime indicates that $\sigma$ and $\mathbf{d}$ are not necessarily "associated" fields, in the sense that the actual strain-rate $d^{\prime}$ that the polycrystal undergoes when the stress field is $\sigma^{\prime}$, has in general no relation to d; e.g. the volume average $\mathbf{D}=\overline{\mathbf{d}}$ and $\mathbf{D}^{\prime}=\overrightarrow{\mathbf{d}^{\prime}}$ are in general different. Hill (1967) has given sufficient conditions in order that Eq. (1) hold, so the no-correlation condition represented by Eq. (1) is now known as Hill's condition. The sufficient conditions given by Hill correspond to what is called a macro-homogeneous situation, i.e. one in which, in particular, the volume averages depend negligibly on the chosen RVE (provided it is indeed representative, thus large enough). Obviously, this is a situation that both theorists and experimentalists would like to enjoy more often. However, a macro-homogeneous field, say the strain-rate d, is something rather realistic (at least in some "useful" part of a deformed test sample), whereas a microhomogeneous field, $\mathbf{d}(\mathbf{x}) \equiv \mathrm{D}$, thus a field $\mathbf{d}$ that would be seen as uniform at the micro-scale, is something highly irrealistic- although it is such field that is considered by the Taylor model. Indeed, any measurement implies a spatial averaging which is done with a certain step $\delta$ (the step $\delta$ gives the "scale" of the measurement) and it thus eliminates those fluctuations whose characteristic distance is smaller than $\delta$. So the macroscopic measurements, which represent averages at the scale of an RVE, involve smoothed-out variations: each time we refine the observation scale, we find more plastic inhomogeneity, until we reach the scale of dislocations which are a "concentrate of plastic inhomogeneity". Bishop and Hill (1951) were plainly aware of the inhomogeneous nature of the fields $\sigma$ and $\mathbf{d}$ in a deformed polycrystal, and in particular they stated (on p. 424) that "it may well be that [the fields $\sigma$ and d] must be allowed to vary continuously, and not be restricted to take constant values within each of a finite number of regions (which may be grains or parts of grains)". 
One may now state more: (i) any piecewise uniform field of strain (or strain-rate) is necessarily a wholly uniform field, together with the associated field of material spin (or spin-rate), if the displacement (or the velocity) is asked to be continuous at the interfaces between the domains where the field is to be constant. The only possible exception is perhaps the case of all planar interfaces (which is clearly a very particular situation for a physical aggregate; even in that situation it would be very difficult to build a piecewise uniform, but not uniform strain field). Although this result may seem surprising, its proof is quick and elementary (Arminjon, 1991b). So no model should be argued to be more exact than Taylor's model, if it assumes a continuous velocity field and a piecewise uniform strain-rate field, because these two conditions, taken together, imply that the complete Taylor model (uniform strain-rate and uniform rate of material spin) must be assumed. If any model predicts one value of strain-rate (and thus one value of stress) for one constituent (e.g. for one grain, in practice often for one crystal orientation), then this value can be interpreted at best as the average value of the strain-rate (or stress) in the constituent. Since this is the case for most models that have ever been used for texture prediction, the interpretation of these models has to be reexamined in order to account for this result.

(ii) Now if one merely asks to a model that it should predict correctly the average values of strain-rate in the different constituents, it will not be trivial to check this correctness. Indeed, suppose one prescribes any thinkable distribution $\left(\mathbf{D}^{k}\right)_{k=1, \ldots, n}$ of the average strain-rates in a bounded aggregate comprising a finite number $n$ of constituents with regular boundaries. Then it is possible to build a regular velocity field $\mathbf{v}$, thus e.g. no sliding at the boundaries, such that the associated field of compatible strain-rate, $d=\operatorname{sym}(\nabla v)$, has the prescribed average values, i.e. the volume average of $\mathbf{d}$ in each constituent $\Omega^{k}$ is the prescribed value $\mathbf{D}^{k}$. The construction is technical (Arminjon, 1991b). Several additional, physically relevant results, have been proved in connection with this in the same paper and in Arminjon (1991a). Here we merely add that the theorem is also true if one substitutes (usual) strain for strain-rate, and displacement for velocity.

In summary, no distinction can be made between different polycrystal models, on the basis of the compatibility of the predicted inhomogeneous distributions of the strain (-rate) among the different constituents. Either one assumes a uniform strain inside each constituent, and then the only model which will produce a compatible strain field is the complete Taylor model- which is undoubtedly a gross approximation, although it has the important property of providing an upper limit, thus one extreme behaviour (the other extreme is provided by the assumption of uniform stress, see below). Or one does not make that assumption of a piecewise uniform strain field, hence interpreting the predicted strains as the average ones in the constituents: then, with every thinkable distribution of the strains, thus predicted by any reasonable or unreasonable model, one can associate a compatible strain field that leads to precisely this distribution of the average values. This is not to say that no physical condition restricts the distribution of the average strains among the different constituents, for the actual distribution is certainly not purely random (and the proposed model indeed assumes a very particular kind of strain distribution). But the notion that the strain in a constituent should be compatible with the strains of the neighbouring constituents has no rigorous consequence on the range of admissible models, within the class of models which predict one strain for one constituent. This conclusion is, we believe, unescapable due to the foregoing mathematically founded arguments. It does not forbid one to use heuristic "compatibility arguments" based e.g. on the "brick" geometry, provided it leads to clearly defined 
models with some predictive capacity (for polyedric constituents, the compatibility of a piecewise uniform strain field does not automatically imply its complete uniformity).

\section{Energy minimization as a criterion to select the inhomogeneous strain distribution}

Under some testing conditions, e.g. rolling or channel-die compression, a homogeneous material, thus e.g. a single crystal (or a polycrystal, but seen at the macroscopic scale), undergoes a "mixed" state of partially imposed stress plus partially imposed strain, instead of a "pure" state of fully imposed strain or fully imposed stress. That is, some components of strain-rate, $D_{i j}$ with $i j \in I J$, as well as the other, "complementary" components of stress, thus $\Sigma_{i j}$ with $i j \notin I J$, are imposed by the boundary conditions. Consider, for instance, the case of frictionless channel-die compression with (1) the compression axis and (3) the channel direction. Then $D_{11}$ (negative), $D_{22}$ and $D_{12}$ (both zero) are imposed, whereas $\Sigma_{13}, \Sigma_{23}$ and $\Sigma_{33}$ are imposed zero, due to the free surface perpendicular to the channel axis (Kocks and Chandra, 1982). (Due to plastic incompressibility, only two of the data $D_{11}, D_{22}$ and $D_{33}$ may be imposed, thus in that case the imposed data $D_{22}=0$ and $D_{11}$ determine $D_{33}=-D_{11}$. Also note that a heterogeneous material, e.g. a bicrystal, subjected to such boundary conditions as e.g. frictionless channel-die compression, will in general not undergo a uniform stress/strain state, hence the foregoing description of the mixed stress/strain state does not apply.) The detailed implications of such mixed conditions for a single crystal obeying the Schmid law were first studied by Renouard \& Wintenberger (1976). In particular, they proved that the crystal must select a set of active slip systems and slip rates such that the difference of the total work-rate and the work-rate of the imposed stresses, is minimal. A result similar to that of Renouard \& Wintenberger (1976) was obtained by Van Houtte (1982), though his work concerned the equivalence between a "minimum internal work" principle and a "maximum external work" principle in the same context, rather than a deduction of either principle from Schmid's law.

Since the virtual work-rate may indifferently be defined in terms of either the virtual slip rates or the associated virtual strain-rate, the result of Renouard and Wintenberger (1976) may be reexpressed in saying that the crystal must select a strain-rate that minimizes the same difference. In particular, when the imposed stresses are zero, the crystal must select a strain-rate $\mathbf{D}^{c}$ that minimizes the work-rate among strain-rates D* whose components $D^{*}$, with $i j \in I J$, have the imposed values, $D^{*}{ }_{i j}=D_{i j}$. This result may be considered as the basis of the relaxed Taylor theory, in the "minimum internal work" form proposed by Van Houtte (1981). [This form was proved by Van Houtte (1982) to be equivalent to the "maximum external work" form, proposed by Mecking (1980) and by Kocks and Canova (1981).] We indeed may say that the relaxed Taylor theory is a model in which one assumes that, at large proportional strains, each constituent crystal of a polycrystal is subjected to a "partially imposed" strain with zero "complementary" stresses. From the considerations in $\$ 2.1$, it follows that such assumption should concern the average strain in the constituent crystal, so we do not find the usual "grain shape argument" very convincing. (According to this argument, flattened grains during rolling at high strains might undergo shear strains in planes perpendicular to the rolling plane (1-2), thus $D_{13}$ and $D_{23}$, at a lower "incompatibility cost" than for a shear in the rolling plane, $D_{12}$. So $D_{13}$ and/or $D_{23}$ might be relaxed, i.e. not be taken in the set of the imposed components.) One may also doubt this argument if one looks at real microstructures: at moderate strains, the strain is already non-uniform, whereas at high strains the grains are fragmented into more or less equiaxed 
domains. It interesting in this context to recall that a "shear relaxation" was proposed in a different way by Leffers (1968), without invoking grain shape arguments, and that it was successfully applied to the simulation of the copper texture. Recently, Van Houtte (1995) has used an upper bound method in a simplified "matrix-inclusion" situation, to assess the "accommodation work" in the matrix, that occurs due to the different strain in the grain and the matrix. In this framework, he has confirmed that it is energetically favourable to have an inhomogeneous strain distribution. Two important points are that (i) he finds this to be true regardless of the grain shape, and (ii) within an upper bound method, a solution that leads to an energetic gain is automatically closer to the exact solution.

Thus the analysis of Schmid plasticity for a crystal subjected to partially imposed strain with zero complementary stresses shows an example where the actual strain-rate minimizes the work-rate done, and the analysis of a matrix-inclusion system shows another example. In both situations, the minimization principle is true regardless of the grain shape. In the second example, the total plastic work-rate must be minimized with respect to the incompressible velocity field $\mathbf{v}^{*}$ satisfying the boundary conditions on the entire boundary. This is in fact a general theorem for plasticity with boundary conditions in velocity (the proof of which is easy) which demands only that the maximum work principle applies to the local constitutive law (Hill, 1950). The incompressibility condition, div $\mathbf{v}^{*}=\operatorname{tr} \mathbf{d}^{*}=0$, is there to ensure that the associated strain-rate, $d^{*} \equiv \operatorname{sym}\left(\nabla \mathbf{v}^{*}\right)$, is indeed a normal to the local yield criterion and thus may be associated with at least one admissible stress tensor $\sigma^{*}$. And the maximum work principle indeed applies to any crystal obeying Schmid's law (Bishop and Hill, 1951). Thus for any crystal aggregate $\Omega$, whether statistically homogeneous or not, the actual velocity field minimizes the global work-rate among velocity fields which are incompressible and satisfy the boundary conditions:

$W_{\text {actual }} \leq W_{\text {virtual }} \equiv \int_{\Omega} \sigma^{*}: \mathbf{d}^{*} d V, \mathbf{d}^{*} \equiv \operatorname{sym}\left(\nabla \mathbf{v}^{*}\right), \sigma^{*}$ and $\mathbf{d}^{*}$ associated by Schmid's law

[As a consequence, the strain-rate (field) in each constituent crystal, taken separately, does not minimize the work-rate done in this constituent.] But to apply directly this result so as to "solve the problem of polycrystal plasticity", we should know the exact boundary conditions that apply to an RVE, which is not possible (e.g. the exact boundary conditions vary from one RVE to its neighbour, whereas we want to solve one problem). And we should consider continuous fields of velocity rather than distributions of strainrates among constituents. But since we want to get a volume-fraction model or "onepoint model", predicting one strain-rate for one constituent, we really must keep a discrete sum over the different constituents. So to deduce a useful model from the general minimization theorem, we need to replace the integral in Eq. (2) by a discrete sum over the different constituents. Here several notions of statistical homogeneity, all needed to make licit the operation of any one-point model, have to be used.

\section{Statistical homogeneity and the justification of using a one-point model}

(i) When we use a micro-macro model, we want to obtain relations between microand macro-scale in a macro-homogeneous situation, thus one in which the macroscopic fields of stress and strain, $\Sigma$ and $\mathbf{D}$, do not vary significantly in space (in practice, this is true only in some "useful part" of specially suited test samples, e.g. the central part of a tension sample, but then the macro-law between $\Sigma$ and $\mathbf{D}$ may be used pointwise 
for any work-piece). Since those macro-fields are just the volume averages of the microfields $\sigma$ and $d$ in an RVE, this is translated into approximate conditions (asymptotically exact in the ideal situation of a space-filling material) on the fields $\sigma$ and $d$. Such conditions were first defined by Hill (1967) and imply, in particular, Hill's no-correlation condition (1). In other words, they hence allow to state that the virtual work-rate spent, per unit volume, by an actual stress field $\sigma^{\prime}$ (thus in particular a self-equilibrated one) and an actual strain-rate field d, not necessarily associated with the former (see above), is simply

$$
W^{*} \equiv \int_{\Omega} \sigma^{\prime}: \mathbf{d} d V / V(\Omega)=\Sigma^{\prime}: \mathbf{D}
$$

Equation (3), together with the maximum work principle (at the micro-scale), implies, among other things, that the macro-law between $\Sigma$ and $\mathbf{D}$ also obeys the maximum work principle (Bishop and Hill, 1951).

(ii) Obviously, a macro-homogeneous situation for the mechanical fields depends on the implicit assumption that the material itself is statistically homogeneous (SH), i.e. two RVE 's $\Omega$ and $\Omega^{\prime}$ should differ only in statistically unsignificant details. To state this assumption precisely, one must first identify which microstructure parameters are relevant to the mechanical behaviour. To do this in an operational way, one just has to list which parameters enter the micro-law, and that list $\mathbf{X}$ we call the "state" for short. Thus for a polycrystal made of crystallites obeying Schmid's law with usual local hardening, we have the crystal orientation $\mathbf{R}$ and the integrated amounts of slip on each slip system, $\gamma^{s}$ with $s=1, \ldots, S$ ( $S$ is the number of independent slip systems,

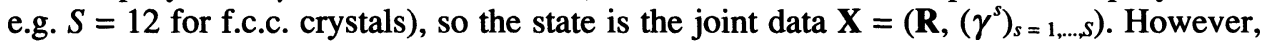
in practice, the amounts of slips will be a function of $\mathbf{R}$ and the past history of deformation (moreover, it is often assumed, and it will indeed be assumed in the applications presented here, that all crystals are equally hardened, to get simple calculations). So here we retain only $\mathbf{R}$ for simplicity. In the case that $\mathbf{R}$ is assumed to vary continuously, the precise definition of an SH material (Arminjon, 1991a) becomes rather transparent: it simply means that the average difference between the ODF's $f$ and $f^{\prime}$ is negligible for any two RVE 's $\Omega$ and $\Omega^{\prime}$ that are large enough [this concerns the statistical homogeneity of the one-point information; the general definition are easy to adapt to the $N$-point information, Arminjon (1991a)]. But here we will consider a discrete orientation distribution, thus a true aggregate of (perfectly oriented) crystals. A precise, though a bit more technical definition of an SH material may be given in that case also (Arminjon, 1991a).

(iii) Let us specialize to one-point micro-macro models. In such models, as already noted, we have one stress and one strain for one "constituent", and it is now evident that what must be called "constituent" in a one-point model is not a unique (connected) geometrical entity, but instead the zone $Z_{X}$ of the material where the state (thus here the crystal orientation $R$ ) has some value $X$. Hence the zone $Z_{R}$ may well contain a lot of non-contiguous crystallites with the same orientation $\mathbf{R}$. And "the" value of e.g. the strain-rate for the orientation $\mathbf{R}$ must obviously be defined as the volume average $\widetilde{\mathbf{d}}(\mathbf{R})$ of the micro-field $\mathbf{d}$ in the zone $\mathrm{Z}_{\mathrm{R}}$. But to take that volume average, we have to select an RVE $\Omega$, i.e. we have to take the average of d over those points in $\Omega$ that have the orientation $\mathbf{R}$ (thus over $\Omega \cap Z_{R}$ ). The question then arises whether we would get the same result if we would take another RVE $\Omega^{\prime}$. Of course, we may only expect that, in some favourable situation, the difference between the two averages is small (if $\Omega$ and $\Omega^{\prime}$ are truly representative). In this situation, precisely defined by Arminjon (1991a), we say that the field d is statistically homogeneous (SH).

(iv) Last requirement of statistical homogeneity: in a one-point model, not only do we 
have one stress and one strain (-rate) for one "constituent" (one orientation $\mathbf{R}$ ), but still these two, which are volume averages of the fields $\sigma$ and $d$ in the zone $Z_{R}$, are assumed to be related together by the micro-law (thus here by Schmid's law). In order that this may be true, the same kind of condition should apply in $Z_{R}$ as it applies at the macro-scale to ensure the existence of a macro-law between $\Sigma$ and $D$. The fields $\sigma$ and $\mathrm{d}$ must hence satisfy Hill's "macro-homogeneity" conditions in the zone $\mathrm{Z}_{\mathrm{R}}$ [in the present case, it is rather a meso-homogeneity, Arminjon (1991b)]. In particular, when two couples of actual fields, $(\sigma, d)$ and $\left(\sigma^{\prime}, \mathbf{d}^{\prime}\right)$, are considered (corresponding to two macroscopic situations, with here "macroscopic" in the usual sense), one should have the following no-correlation condition for every orientation $\mathbf{R}$ :

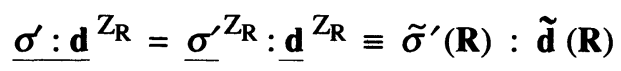

where the symbol ${ }^{-Z_{R}}$ means volume average in the zone $Z_{R}$. To simplify these notations, let us assume that our polycrystal (or rather our RVE $\Omega$ in our polycrystal) contains $n$ different crystal orientations $\mathbf{R}_{1}, \ldots, \mathbf{R}_{n}$, denote $\Sigma^{\prime k}(k=1, \ldots, n)$ the average value of $\sigma^{\prime}$ in $\Omega^{k} \equiv \Omega \cap Z_{R_{k}}$ and the like for $\mathbf{d}$. Thus we must have for every orientation $k$ :

$$
\int_{\Omega^{k}} \sigma^{\prime}: \mathbf{d} d V / V\left(\Omega^{k}\right)=\Sigma^{\prime k}: \mathbf{D}^{k}
$$

In combining (3) and (5), we get (using the fact that $\int_{\Omega}=\int_{\Omega^{1}}+\ldots+\int_{\Omega^{n}}$ ):

$$
W^{*}=\Sigma^{\prime}: \mathbf{D}=f_{1} \Sigma^{\prime \prime}: \mathbf{D}^{1}+\ldots+f_{n} \Sigma^{\prime n}: \mathbf{D}^{n}, f_{k} \equiv V\left(\Omega^{k}\right) / V(\Omega)
$$

$\left(f_{k}\right.$ is the volume fraction of orientation $\mathbf{R}_{k}$, thus $f_{1}+\ldots+f_{n}=1$ ). So we have indeed replaced the integral expression of the virtual power (3) by a discrete sum, in which the $k$ term is the virtual power spent in orientation $k$. [But we did not do that for expression (2), which applies to virtual fields, and indeed expression (2) does not reduce in general to a sum like (6).] Furthermore, the average stress and average strain-rate in $\mathbf{R}_{k}, \Sigma^{k}$ and $\mathbf{D}^{k}$, are related by the local constitutive law, thus by Schmid's law.

\section{The Taylor-Bishop-Hill upper bound (revisited)}

Equation (6) applies in particular to the case where $\sigma^{\prime}=\sigma$ is indeed the actual stress field that occurs in the material when the strain-rate field is d. It thus gives the actual work-rate per unit volume at the macro-scale, as the weighted averaged of the volume work-rates spent in the different "constituents" (crystal orientations):

$$
W(\mathbf{D})=\Sigma: \mathbf{D}=f_{1} \Sigma^{1}: \mathbf{D}^{1}+\ldots+f_{n} \Sigma^{n}: \mathbf{D}^{n}=f_{1} W^{1}\left(\mathbf{D}^{1}\right)+\ldots+f_{n} W^{n}\left(\mathbf{D}^{n}\right)
$$

[It has been accounted for the fact that, due to the maximum work principle, the Schmid law makes the work-rate, though not the stress, depend uniquely on the strain-rate $\mathbf{D}^{k}$, $W^{k}=W^{k}\left(\mathbf{D}^{k}\right)$.] From the definition of $\Sigma$ as $\bar{\sigma}$ and that of the actual average stress $\Sigma^{k}$ in $\Omega^{k}$, we get:

$$
\Sigma: \mathbf{D}=\left[\int_{\Omega} \sigma d V / V(\Omega)\right]: \mathbf{D}=\left[\left(\int_{\Omega^{1}} \sigma d V+\ldots+\int_{\Omega^{n}} \sigma d V\right) / V(\Omega)\right]: \mathbf{D}=f_{1} \Sigma^{1}: \mathbf{D}+\ldots+f_{n} \Sigma^{n}: \mathbf{D}
$$

Let us consider, in every constituent $k$, a stress $\Sigma_{\text {sup }}{ }^{k}$ that is associated, by Schmid's 
law, to the macroscopic strain-rate D. By the maximum-work principle, we have $\Sigma^{k}$ : D $\leq \Sigma_{\text {sup }}^{k}: \mathbf{D}=W^{k}(\mathbf{D})$, hence we obtain by $(8)$ :

$$
W(\mathbf{D})=\Sigma: \mathbf{D}=f_{1} \Sigma^{1}: \mathbf{D}+\ldots+f_{n} \Sigma^{n}: \mathbf{D} \leq f_{1} W^{1}(\mathbf{D})+\ldots+f_{n} W^{n}(\mathbf{D}) \equiv W_{\text {sup }}(\mathbf{D})
$$

This is the Taylor-Bishop-Hill upper bound. However, it has not been assumed here that the stress and strain-rate fields are uniform within a given constituent (orientation), and yet, due to the assumptions of statistical homogeneity, a discrete sum has been obtained instead of an integral.

\section{Extreme energy minimization: the lower bound model and the Sachs-type models}

It is well-known that a lower bound is obtained if one assumes that the stress is the same for each crystal orientation: this was proved by Bishop and Hill (1951, p. 425). It is also known that this assumption (the "static" model) is different from the model proposed by Sachs (1928), according to which the stress is uniform up to an orientationdependent multiplicative factor such that the critical shear stress is reached on the bestoriented glide system(s) of the considered crystal orientation. The static model predicts that, in general, only the "weakest point of the aggregate" (Bishop and Hill, 1951), i.e. the weakest grain orientation for the considered stress, will reach the yield point. This is obviously different from Sachs' assumption, hence the latter does not really provide a lower bound. On the other hand, the static model is clearly an unreasonable model, except for the very beginning of plastic strain: one cannot hope to correctly model deformation textures with one grain deformed! (however, it is fair to say that this would not be necessarily the same grain along the deformation, in the case that the grain would rotate towards a "harder" orientation). Thus it is likely that the Sachs model, not the static model, represents an "equivalent of crudeness" in the opposite direction to the Taylor model. As noted by Leffers and Juul Jensen (1991), the Sachs model, when interpreted in the usual way, "does not fulfil the continuum requirement (neither in strain or stress)". But, according to the interpretation proposed here for onepoint models, the strains and stresses "in the constituents" predicted by those models are just the average values, in the constituents, of fields that are heterogeneous in any constituent (though "meso-homogeneous" in the sense outlined hereabove). If one accepts this interpretation, then the above criticism (which would indeed apply to any intermediate model between the static model and the Taylor model) can be overcome. Nevertheless, by modifying Sachs' model through the introduction of random stresses, Leffers (1968) and Pedersen and Leffers (1987) could obtain a significantly better simulation of the brass texture as compared with the original Sachs model (and still more if one compares with the Taylor model), indeed they obtained a correct simulation [see also Leffers and Juul Jensen (1991)]. Probably, the addition of random stresses makes the model less extreme in two different ways: it enhances the power spent (as compared with the Sachs model), and also the randomness, in itself, leads to less sharp textures.

Now we indicate how the lower bound appears in our formulation. The actual power $W(\mathrm{D})$ is the weighted average (7) of the powers corresponding to the actual average strain-rates in the constituents, $\mathbf{D}^{\mathrm{k}}(k=1, \ldots, n)$. On the other hand, the weighted average of that actual average strain-rates themselves is the macroscopic strain-rate:

$$
f_{1} \mathbf{D}^{1}+\ldots+f_{n} \mathbf{D}^{n}=\mathbf{D}
$$


Hence, if we minimize the weighted average of the powers under the mere constraint that the weighted average of the strain-rates is $\mathbf{D}$, we get a lower bound for the actual power:

$$
\begin{gathered}
W(\mathbf{D}) \geq W_{i \mathrm{nf}}(\mathbf{D}) \equiv \\
\equiv \operatorname{Min}\left\{f_{1} W^{1}\left(\mathbf{D}^{*_{1}}\right)+\ldots+f_{n} W^{n}\left(\mathbf{D}^{* n}\right) \text { under constraint } f_{1} \mathbf{D}^{*_{1}}+\ldots+f_{\mathrm{n}} \mathbf{D}^{*_{\mathrm{n}}}=\mathbf{D}\right\}
\end{gathered}
$$

It turns out that this lower bound does correspond to the static model, i.e. if one has the (or any) distribution of strain-rates, $\left(D_{\text {inf }}\right)_{k=1, \ldots, n}$, that reaches the minimum (11), then the stresses $\Sigma_{\text {inf }}{ }^{k}$ which are associated to the $\bar{D}_{\text {inf }}{ }^{k}$ 's by the local constitutive law are all the same. This has been proved in the general context of a local behaviour deriving from a regular convex potential by Arminjon (1991a). This proof does not strictly apply to rate-independent plasticity, however, because the latter does not lead to an everywhere continuous dependence of the stress as function of the strain-rate. A careful examination of this point is left to a future work, because the proposed model does not actually need that the lower bound $W_{\text {inf }}(\mathbf{D})$ indeed correspond to uniform stresses.

We emphasize that, up to know, we have just started clearly (at least, we tried to do so) some assumptions which should be common to all one-point polycrystal models.

\section{The proposed model}

Let us introduce the average inhomogeneity of a possible distribution $\left(\mathbf{D}^{* k}\right)_{k=1, \ldots, n}$ of the (average) strain-rates in the different constituents $k=1, \ldots, n$ :

$$
h=h\left(\left(\mathbf{D}^{* k}\right)\right) \equiv f_{1}\left\|\mathbf{D}^{*_{1}}-\mathbf{D}^{*}\right\|+\ldots+f_{n}\left\|\mathbf{D}^{*_{n}}-\mathbf{D}^{*}\right\|
$$

with $D^{*}$ the weighted (macroscopic) average of those strain-rates:

$$
\mathbf{D}^{*} \equiv f_{1} \mathbf{D}^{*_{1}}+\ldots+f_{n} \mathbf{D}^{*_{n}}
$$

(and where $\|\mathbf{T}\| \equiv\left[\operatorname{tr} \mathbf{T}^{2}\right]^{1 / 2} \equiv\left[T_{i j} T_{i j}\right]^{1 / 2}$ is the Euclidean norm of a tensor $\mathbf{T}$ ). Consider, for a given positive number $r$ and for a given tensor $\mathbf{D}$, the following minimum problem:

$W_{r}=W_{r}(\mathbf{D}) \equiv \operatorname{Min}\left\{f_{1} W^{1}\left(\mathbf{D}^{* 1}\right)+\ldots+f_{n} W^{n}\left(\mathbf{D}^{* n}\right)\right.$ under constraints $\mathbf{D}^{*}=\mathbf{D}$ and $\left.h \leq r\right\}$

Obviously, $W_{r}$ is a decreasing function of $r$. For $r=0$, the solution is the uniform distribution, $\mathbf{D}^{* k}=\mathbf{D}$ for all $k$, and the value $W_{0}$ of the minimum is hence just the Taylor-Bishop-Hill upper bound [Eq. (9)], thus $W_{0}=W_{\text {sup. }}$ On the other hand, once $r$ reaches the inhomogeneity value $R=R(D)$ corresponding to the lower bound, $R \equiv$ $h\left(\left(\mathrm{D}_{\text {inf }}{ }^{k}\right)\right)$, then $W_{r}$ remains equal to the lower bound [Eq. (11)], thus $W_{r}=W_{\text {inf }}$ for $r \geq R^{2}$. So

$$
W_{\text {inf }}=W_{r} \leq W \leq W_{0}=W_{\text {sup }}
$$

\footnotetext{
${ }^{2}$ If it happens that several distributions $\left(\mathrm{D}_{1}{ }^{k}\right),\left(\mathrm{D}_{2}{ }^{k}\right), \ldots$ with different inhomogeneities $R_{1}, R_{2}, \ldots$ give the lower bound (11), then we take the $R$ the infimum of $R_{1}, R_{2}, \ldots: R$ also must be the inhomogeneity of some distribution $\left(\mathbf{D}_{\text {inf }}^{k}\right)$ such that $f_{1} W^{1}\left(\mathbf{D}_{\text {inf }}^{1}\right)+\ldots+f_{n} W^{n}\left(\mathbf{D}_{\text {inf }}^{n}\right)=W_{\text {inf }}(\mathbf{D})$ (this may be proved by a "compacity argument").
} 
Hence, there exists a value $r_{0}$, which a priori depends on $\mathbf{D}$, and with $0 \leq r_{0}(\mathbf{D}) \leq$ $R(D)$, such that the actual power $W$ is equal to the minimum $W_{r}$. Moreover, this value $r_{0}$ is unique, because $W_{r}$ is actually a strictly decreasing function of $r$ for $0 \leq r \leq$ $R$ (Arminjon et al., 1994). For this same reason, the minimum $W_{r}(\mathbf{D})$ is, for $0 \leq r$ $\leq R$, always reached by a strain-rate distribution $\left(\mathbf{D}_{r}^{k}\right)$ that has exactly the inhomogeneity $r$. As a consequence, the inequality constraint $h \leq r$ may be replaced by an equality constraint in the minimization problem (14), $h=r$ (once the value that gives the lower bound, more precisely the smallest possible value $R$ giving the lower bound, has been determined).

Here again, the existence of the number $r_{0}(\mathbf{D})$ depends only on very general assumptions: the assumptions of statistical homogeneity, plus the fact that the microlaw obeys the maximum work principle, or more generally derives from a convex potential. The particular model proposed depends on two assumptions that have a very different status from each other. First, no available framework enables us to predict the value of $r_{0}(\mathbf{D})$ from microscopic data. So to determine the dependence $r_{0}=r_{0}(\mathbf{D})$, we must have recourse to a phenomenological assumption. In the two applications developed so far (Arminjon et al., 1994, for determining the macro-law of reinforced mortars, and the present work, for predicting the deformation textures of steels), we have found that the simplest assumption, $r_{0}(\mathrm{D})=$ Const. $=a$ (for $\|\mathrm{D}\|=1$, thus $r_{0}(\mathrm{D})$ $=a\|\mathrm{D}\|$ if $\|\mathrm{D}\|$ varies), is sufficient. Some indications on what could be done otherwise have been given by Arminjon et al. (1994). Thus at present, we determine a value $a$ that gives a correct prediction for one macroscopic strain-rate $D_{1}$, and then we assume this same value for other tensors $D$, i.e we assume,$r_{0}(\mathbf{D})=$ Const. $=a$ (for $\|D\|$ =1).

\section{The principle of minimal inhomogeneity}

We turn to the second, essential assumption. The parameter $r_{0}$ (depending a priori on D) is, by definition, such that

$$
W(\mathbf{D})=W_{r_{0}}(\mathbf{D})
$$

where $W_{r_{0}}(\mathrm{D})$ is the minimum (14) (with the relevant value $r_{0}$ in the place of $r$ ). Thus, the distribution $\left(\mathbf{D}_{r_{0}}{ }^{k}\right)$ that gives the minimum must satisfy

$$
W(\mathbf{D})=f_{1} W^{1}\left(\mathbf{D}_{r_{0}}{ }^{1}\right)+\ldots+f_{n} W^{n}\left(\mathbf{D}_{r_{0}}{ }^{n}\right)
$$

On the other hand, the actual power is the weighted average of the powers spent in the different constituents [Eq. (7)]:

$$
W(\mathbf{D})=f_{1} W^{1}\left(\mathbf{D}^{1}\right)+\ldots+f_{n} W^{n}\left(\mathbf{D}^{n}\right)
$$

Moreover, the weighted average of both distributions: the actual one, $\left(D^{k}\right)$, and that one which is the solution of the minimum problem (14), is the macroscopic strainrate $\mathbf{D}$ [Eq. (10), and Eq. (13) with $\mathbf{D}^{*}=\mathbf{D}$ from the constraint in Eq. (14)]. At first sight, it seems hence obvious that the distribution $\left(\mathbf{D}_{r_{0}}{ }^{k}\right)$ is the actual distribution, or at least (if the minimum is reached by several distributions) that the actual distribution must be one solution to the minimum problem (14). But this is not necessarily the case. That this is indeed the case, is the basic physical assumption of the model. This 
assumption does not play any rôle for the micro-to-macro transition, but it is necessary for the macro-to-micro transition- and thus for the prediction of deformation textures. Now why is this an assumption, and why then a physical assumption? If the actual distribution of strain-rates is a solution to the minimum problem (14) for the relevant value $r=r_{0}$, i.e. that one for which Eq. (16) is true, then it must satisfy the constraint $h \leq r_{0}$, in fact it even must satisfy $h=r_{0}$, as noted previously. Hence, the necessary assumption is that the relevant value $r_{0}$ is precisely the inhomogeneity of the actual distribution of strain-rate. It thus implies that the value $r_{0}$ has a clear physical meaning. This assumption may be reexpressed as the following equivalent principle (Arminjon et al., 1994): among those distributions of the strain-rate between the constituents that lead to the actual macroscopic strain-rate $\mathbf{D}$ and that spend the same power as the power actually spent to have this strain-rate $\mathbf{D}$, the actual distribution has the least inhomonegenity. One still may say that the inhomogeneity occurs only in so far as it allows to reduce the energy consumption.

Note that the formulation of the principle is in terms of the proposed notion of "constituent-averaged" fields of stress and strain (-rate), i.e. here the average value of stress and strain-rate for a given crystal orientation. It would not make sense to formulate this principle in terms of the space-dependent microscopic fields of stress and strain, for in the latter case we have a minimization theorem, which demands that one restricts to (velocity) fields satisfying the boundary conditions, assumed known- which is impossible in practice [see around Eq. (2)]. Thus the principle makes sense in highly "stochastic" situations for which the exact boundary conditions do not really matter, as long as one wishes only to access to those "constituent-averaged" fields- a very statistical output. This principle of minimum inhomogeneity may be uniquely formulated for any physical behaviour for which a convex potential exists that has a clear interpretation as an energy. In particular, it makes sense for standard plasticity (as here), and also for pure elasticity, elastoplasticity and viscoplasticity. This physical principle does not seem to be reducible to (deducible from) the laws of deterministic mechanics. Instead, it may be seen as similar to a general principle in statistical mechanics, according to which the equilibrium states are those for which the largest possible number of equivalent realizations exist.

\section{NUMERICAL IMPLEMENTATION}

\section{The algorithm for solving the minimum problem}

We have to solve the convex minimum problem (14) involving one linear constraint, $\mathbf{D}^{*}=\mathbf{D}$ with $\mathbf{D}^{*}$ the weighted average (13), and one convex constraint, $h \leq r$ with $h$ given by Eq. (12). The algorithm used now is different from that described by Arminjon et al. (1994), which was used for fiber-reinforced mortars. In the new algorithm, the constraint $\mathbf{D}^{*}=\mathbf{D}$ is utilized (and thus accounted for) to eliminate the strain-rate of the last constituent $(k=n)$ :

$$
\mathbf{D}^{* n}=\left(\mathbf{D}-\sum_{k=1}^{n-1} f_{k} \mathbf{D}^{*}\right) / f_{n}
$$

so that the list of the optimization variables is $\mathbf{Y}=\left(\mathbf{D}^{* k}\right)_{k=1, \ldots, n-1}$. The function to minimize is written as:

$$
F(\mathbf{Y})=\sum_{k=1}^{n} f_{k} W^{k}\left(\mathbf{D}^{*^{k}}\right)=\sum_{k=1}^{n-1} f_{k} W^{k}\left(\mathbf{D}^{*^{k}}\right)+W^{n}\left(\mathbf{D}-\sum_{k=1}^{n-1} f_{k} \mathbf{D}^{*^{k}}\right)
$$


and the inhomogeneity is reexpressed as a function of the list $\mathbf{Y}$ :

$$
h(\mathbf{Y})=\sum_{k=1}^{n-1} f_{k}\left\|\mathbf{D} *^{k}-\mathbf{D}\right\|+\left\|\sum_{k=1}^{n-1} f_{k}\left(\mathbf{D}^{*^{k}}-\mathbf{D}\right)\right\|
$$

At this stage (solution of the minimization problem), the "crystallographic" $W^{k}$ function (which is the same for all crystals up to a rotation) was replaced by a function of the form $\left[Q\left(\mathbf{D}^{k}\right)\right]^{1 / 2}$ with $Q$ a quadratic form that represents the least squares fitting of the square of the actual $W^{k}$ function. The incompressibility condition means that only five independent components have to be retained for each strain-rate $\mathbf{D}^{* k}$, and these are $D_{11}, D_{22}, D_{23}, D_{13}$ and $D_{12}$ (the $D_{33}$ component, when needed, is calculated as $D_{33}$ $=-D_{11}-D_{22}$ ).

Recall that the constraint $h \leq r$ may be replaced by the equality constraint, $h=r$, and this is actually done. However, the problem with equality constraint will only give the correct minimum (14) if $r$ is not greater than the [largest] inhomogeneity $R^{\prime}$ giving the lower bound, and $R^{\prime}$ is not known in advance. For $r>R^{\prime}$, the minimum obtained with $h=r$ becomes an increasing function of $r$ [it is constant for $R \leq r \leq$ $R^{\prime}$, if $R \neq R^{\prime}$ ]. Hence, $R$ the [least] value of $r$ that gives the lower bound, may be determined as giving the least value of $W_{r}^{\prime}(\mathbf{D})$, with $W^{\prime}{ }_{r}(\mathrm{D})$ the minimum obtained with $h=r$. Once this has been done, if we take any value $r \leq R$ [or even $r \leq R^{\prime}$ ], then we know that the solution obtained with $h=r$ is correct. In practice, $R$ depends relatively little on $\mathbf{D}$ and moreover the relevant value $r_{0}(\mathbf{D})$ is significantly smaller than $R$. The constraint $h=r$ may be written as $C(\mathbf{Y}) \equiv h(\mathbf{Y})-r=0$, and this is accounted for by the penalty method. Thus we select a large "penalty coefficient" $\rho$, here $\rho=10^{4}$, and we search for the minimum of the function

$$
F_{1}(\mathbf{Y}) \equiv F(\mathbf{Y})+\rho C(\mathbf{Y})^{2}
$$

without any constraint. Generically, the minimum of $F_{1}$ should be reached for a unique value $\mathbf{Y}_{0}$, but a more detailed study is deferred until later. The minimization method is the method of the conjugate gradient, which is standard. However, this method manages only the choice of a new "descent direction" $\mathbf{Z}_{m+1}$, such that the $(m+1)$ th approximation of $\mathbf{Y}_{0}$ is given by

$$
\mathbf{Y}_{m+1}=\mathbf{Y}_{m}+\lambda \mathbf{Z}_{m+1}
$$

for some value $\lambda$. The latter is determined by a specific algorithm for unidirectional minimization. We use the Newton method combined, in case it fails to converge, with a kind of dichotomy (though with three points!) which ensures that, at least, $F_{1}\left(\mathbf{Y}_{m+1}\right)$ $<F_{1}\left(\mathbf{Y}_{m}\right)$. The convergence test is double: one checks that (i) the Euclidean norm of the gradient of the function to be minimized is lower than some tolerance, $\left\|\nabla F_{1}\right\| \leq$ $\varepsilon^{\prime}=N \varepsilon$ [with $N=5(n-1)$ the number of minimization variables, here $N=2089 \times 5$ $=10445$, see below], and (ii) the maximum difference between any component of the retained solution vector $\mathbf{Y}_{M}$ and the same component of the vector $\mathbf{Y}_{M-p}$ corresponding to some past, rather distant iteration, is lower than some other tolerance $\eta$. In this work, the following values were adopted: $\varepsilon=10^{-6}, \eta=10^{-2}$ and $p=100$. The initial vector $\mathbf{Y}_{m=1}$ was obtained by random perturbation of the vector corresponding to the Taylor model, $\mathbf{Y}_{\text {Taylor }} \equiv(n-1)$ times $\mathbf{D}$. For a given number $r$, small as compared with the value $R$ giving the lower bound, and for a given tensor $D$, the program runs some 4 to 5 hours on a PC $486 / \mathrm{DX} 2 / 66 \mathrm{MHz}$ computer to get the solution vector $\mathbf{Y}_{M}$, 
with these parameters. But when $r$ increases so that many "grains" $k$ undergo practically zero strain-rate, $\mathbf{D}_{r}{ }^{k} \approx 0(r \geq 0.5$, see below $)$, it is not always possible to reach the tolerance $\varepsilon^{\prime}$ for $\left\|\nabla F_{1}\right\|$. This is because $\nabla W^{k}=\Sigma^{k}$ is discontinuous at $\mathbf{D}^{k}=0$, hence the minimum of $F_{1}$ corresponds in such cases to a point $Y_{0}$ such that $\nabla F_{1}\left(Y_{o}\right)$ is not exactly zero.

\section{Physically relevant aspects of the computation procedure}

As for the work by Arminjon and Donadille (1990) and Arminjon and Imbault (1993, 1994), which was based on a different polycrystal model in the actual computations, the plastic deformation of bcc crystals was assumed to occur by "pencil-glide" in <111> directions, using the method proposed by Royer et al. (1979). Apart from the determination of the strain-rate distribution, which is characteristic from the polycrystal model and has just been described for the present model, the same computation procedure was used for texture prediction as the procedure used in those previous works [except for that part in Arminjon and Imbault $(1993,1994)$ which concerns the continuity equation for the ODF, not used here]. In particular, the lattice spin-rate tensor $\Omega=$ $\boldsymbol{\Omega}(\mathbf{D}, \mathbf{R})$ (the antisymmetric tensor such that $\mathbf{R}=\boldsymbol{\Omega} . \mathbf{R}$ ) is calculated once and for all, for a regularly spaced orientation network in Euler's space $\left(10^{\circ}\right.$ for each angle: $\varphi_{1}$ from $0^{\circ}$ to $180^{\circ}, \Phi$ from $-5^{\circ}$ to $95^{\circ}, \varphi_{2}$ from $0^{\circ}$ to $90^{\circ}$, thus $n=19 \times 11 \times 10=$ 2090 orientations). After this preliminary step has been completed, to calculate the evolution of any set of orientations, the value of $\Omega$ for the relevant orientation $\mathbf{R}$ is obtained by linear interpolation. In the new model, however, the different orientations $\mathbf{R}_{k}$ are coupled together, in the sense that their volume fractions $f_{k}$ and thus the texture field do have an influence on the $\Omega$. But in this work, we have considered an isotropic texture when calculating the field $\Omega$. This has been done in putting the weight $f_{k}=$ $\sin \Phi_{k}$ on the orientation $\mathbf{R}_{k}\left(\varphi_{1 k}, \Phi_{k}, \varphi_{2 k}\right)$ of the regular network. Thus, the variation of the field $\Omega$ with the texture has been neglected. Previous work has indeed shown that rather accurate texture predictions can be obtained for quite different initial textures with one and the same rotation field.

The field $\Omega$ being calculated for an isotropic texture, it is an isotropic function of its arguments, i.e. the macroscopic strain-rate $\mathbf{D}$ and the crystal orientation $\mathbf{R}$ (Arminjon and Imbault, 1993). This means that, for any rotation tensor Q:

$$
\Omega\left(\mathbf{Q} . \mathbf{D} \cdot \mathbf{Q}^{\mathrm{T}}, \mathbf{Q} \cdot \mathbf{R}\right)=\mathbf{Q} \cdot \mathbf{\Omega}(\mathbf{D}, \mathbf{R}) \cdot \mathbf{Q}^{\mathrm{T}}
$$

This allows us, in the long preliminary step, to calculate $\Omega(\mathbf{D}, \mathbf{R})$ for diagonal tensors D only:

$$
\mathbf{D}=\operatorname{diag}(1,-q, q-1) \equiv \Delta(q) \quad(0 \leq q \leq 0.5+\delta q),
$$

thus 6 values of $q$ for an interval $\delta q=1 / 8$ (interpolating then also with respect to $q$ to get $\Omega$ at the stage of texture prediction). Moreover, since we take $\Omega$ as a function of $\mathbf{D}$ and $\mathbf{R}$ only, we have to specify the axes relative to which $\Omega$ measures the rate of rotation of the crystal axes. As before, these axes have been here those that rotate with the same spin rate $\mathbf{W}$ as the RVE- and for the deformations studied here, these were simply the sheet axes (rolling direction, transverse direction, normal direction). (And we thus select those axes as the reference axes, so $\mathbf{W}=0$.) These axes are convenient, for we assume that every constituent undergoes the same material spinrate, and thus also the same spin-rate as the RVE: $\mathbf{W}^{k}=\mathbf{W}$ for all $k$. This assumption 
is enforced in our model, at least for a rigid-plastic behaviour, since the spin-rate, as an antisymmetric tensor, does not enter the power function of a constituent. ${ }^{3}$

Then, the calculation of the deformation texture for an imposed deformation path follows a set of $n^{\prime}=1800$ orientations $\mathbf{R}_{k}$. This set has been initially selected by quasirandom generation so as to represent an isotropic texture when affected with identical weights. To do this, the angle $\Phi$ was selected with a probability proportional to sin $\Phi$, thus a uniform law for $\cos \Phi, \varphi_{1}$ and $\varphi_{2}$. When an initial texture is present, it is accounted for in affecting each orientation $\mathbf{R}_{k}$ with the value of the initial ODF $f_{0}$ for the initial orientation $\mathbf{R}_{k}{ }^{0}$, hence

$$
f_{k}=f_{0}\left(\mathbf{R}_{k}^{0}\right) /\left[f_{0}\left(\mathbf{R}_{1}^{0}\right)+\ldots+f_{0}\left(\mathbf{R}_{n^{0}}{ }^{0}\right)\right]
$$

After the final orientations have been computed, a continuous ODF $f$ is obtained by setting a Gaussian spread on each orientation, here $\phi_{0}=6^{\circ}$ or $9^{\circ}$, and calculating the corresponding coefficients on the basis of generalized spherical harmonics (Bunge, 1969), for even values of $l$ [see Arminjon and Donadille (1990) for some justification on this point], and with the truncation order $l_{\max }=22$. In this work, cubic-orthotropic symmetry has been assumed at the stage of the actual ODF calculation, consistently with the imposed deformations. The default deformation step was $\delta \varepsilon=0.025$, but in some cases it was necessary to reduce the step so as to reach the imposed accuracy when extracting the Euler angles from the updated rotation matrix (this is due to the "explicit" scheme adopted for updating the rotation matrix, $\mathbf{R}^{\prime}=\mathbf{R}+\delta \varepsilon \Omega(\mathbf{D}, \mathbf{R}) . \mathbf{R}$, which gives only an approximately orthogonal matrix $\mathbf{R}^{\prime}$; due to the rate-independence, time is identified with strain).

\section{RESULTS AND DISCUSSION}

\section{Predicted inhomogeneity of the strain-rate and stress distributions}

The strain-rate distribution $\left(\mathbf{D}_{r}^{k}\right)_{k-1, \ldots, n}$ was calculated for several values of the inhomogeneity parameter $r$ [Eqs. (12) and (14)] and for the $\left(N_{q}+1\right)$ diagonal tensors $\mathbf{D}=\Delta\left(q_{i}\right)$ [Eq. (25)], with $q_{i}=(i-1) \times \delta q\left(i=1, \ldots, N_{q}+1\right)$. In a first step, a smaller set of regularly distributed orientations $(n=462$, with weights $\sin \Phi$ corresponding to an isotropic texture) and only two tensors $\mathbf{D}$ were considered, but a number of different values were taken for $r$, so as to get a tendency for the distributions and to have an idea of the zone corresponding to the lower bound $(r=R)$. It was found that $R$ is larger than 1 (Figure 1 shows this for $q=0$, which corresponds to rolling deformation in direction 1 and in the plane 1-2). The stress inhomogeneity decreases as the

\footnotetext{
${ }^{3}$ Perhaps it is imposed, more generally, by the use of a true one-point model (no microgeometry). As noted by Leffers et al. (1995) and by Van Houtte (1995), models with some microgeometry may describe non-uniform material rotation (in their works, these are "inclusion-matrix" configurations). A model with non-equal material rotation of neighbouring crystals may be obtained, in the proposed framework, if one defines the "constituents" to be some cells, each of which containing several crystals. The power function of each cell is obtained by periodic homogenization, and then the proposed model is applied to find the distribution of the average strain-rates between the different cells. Such a "twoscales micro-macro model" has been proposed by Arminjon et al. (1994), and applied to fiber-reinforced mortars, which are strongly inhomogeneous materials.
} 


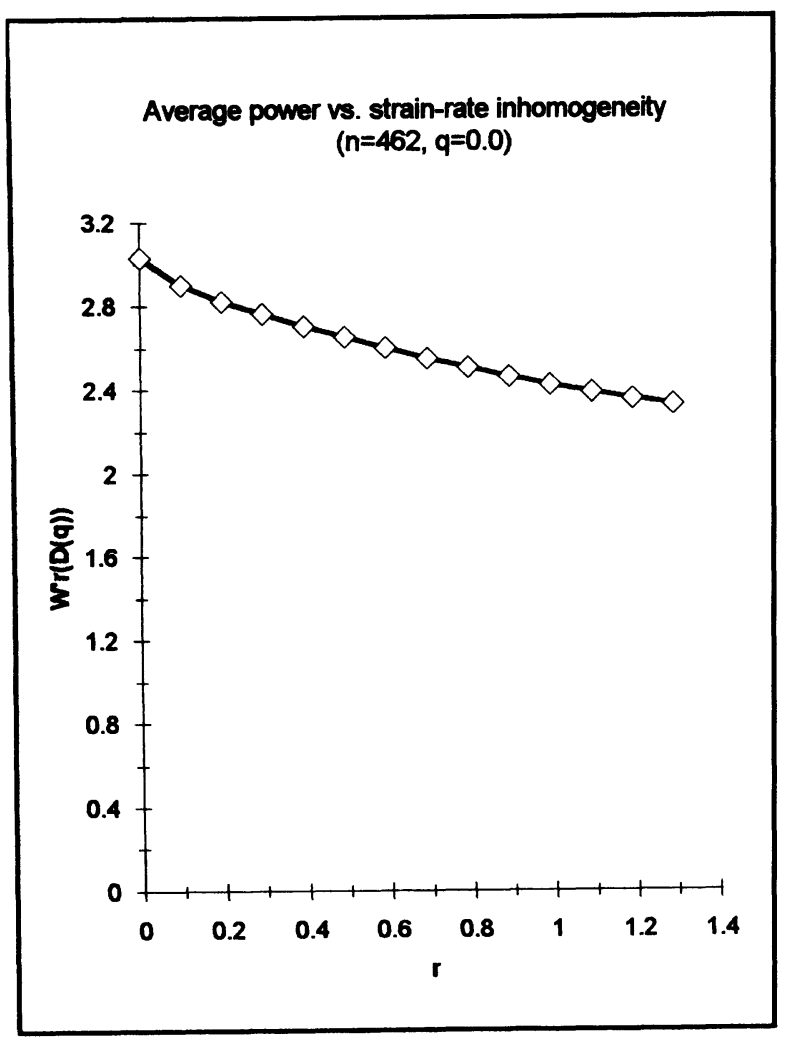

Figure 1 Dependence of the minimum average power, given by Eq. (14), on the value $r$ of the average inhomogeneity in strain-rate.

strain-rate inhomogeneity $r$ increases, as expected (Figure 2), but it does not seem to evanesce as $r$ tends towards $R$ (cf. the above discussion of the lower bound model). Whereas the value of the minimum $W_{r}$ decreases smoothly for $0 \leq r \leq R$ (Figure 1), the individual strain-rate inhomogeneities (the average value of which is constrained to be the given value $r$ ) evolve drastically with $r$. At small values of $r$, the individual values of any strain-rate component are regularly distributed around the mean (macroscopic) value ( $r \leq 0.3$ on Figure 3 for $D_{11}$, the major macroscopic component). As $r$ is increased, certain "grains" (orientations) undergo large strain-rates. Beyond some value ( $r=0.5$ here), these "soft" grains undergo even a larger strain-rate, and moreover some other grains are practically undeformed. The number of the extremely deformed grains, as well as the number of the undeformed ones, increases with $r$.

Since a situation with undeformed grains seems rather unrealistic from what we know about microstructures of deformed low-carbon steels [e.g. Kern and Bunge (1984)], full calculations (for $n=2090$ regularly distributed orientations with weights $\sin \Phi$ and for $\left(N_{q}+1\right)=6$ tensors D) were done for two small values of $r: 0.20$ and 0.25 . Figure 4 shows the histograms of the inhomogeneity in norm and those of the inhomogeneity 


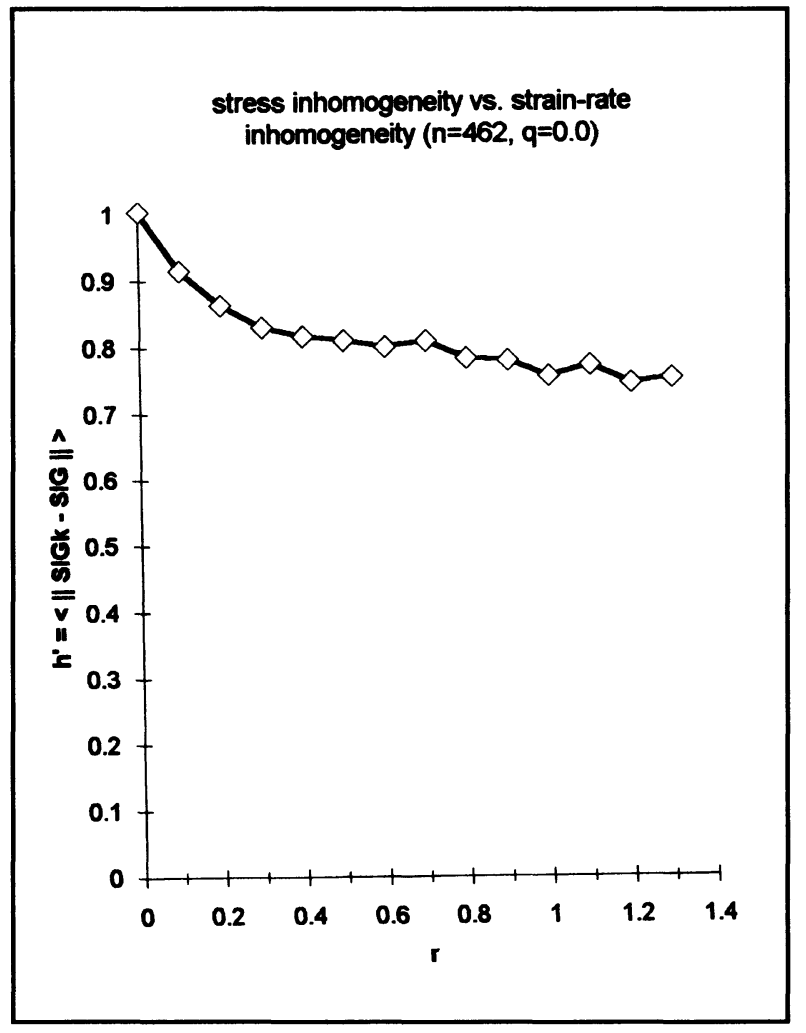

Figure 2 Dependence of the average inhomogeneity in stress on the value $r$ of the average inhomogeneity in strain-rate.

of the 5 independent components, at $r=0.25$, for $q=0$ (rolling). Figure 5 shows the same, except that $q=0.5$ (which corresponds to uniaxial tension for an isotropic material). In these histograms, e.g. for the $D_{i j}$ component, the height $H(a, b)$ of each column represents the volume fraction of that orientations in the regular network with uniform texture (thus with weights $\sin \Phi$ ) for which the inhomogeneity is in the range $[a, b]$ corresponding to the column. Thus :

$H_{i j}(a, b)=\left(\right.$ Sum of $\sin \Phi_{k}$ for orientations $k$ with $\left.a<D^{k}{ }_{i j} D_{i j}<b\right) /\left(\right.$ Sum of $\left.\sin \Phi_{k}\right)$

It is seen that the distribution of the inhomogeneities is rather regular and symmetrical for the shear components: in several cases, e.g. $D_{12}$ and $D_{23}$ for rolling, the distribution looks like a Gaussian one (this is also true for the transverse strain-rate in rolling $D_{22}$ ). The diagonal components are in general less regularly distributed, e.g. not symmetrical. This is true in particular for that component $\left(D_{11}\right)$ which has the greatest average, as also for $D_{33}$ (not shown). Less regular histograms are also obtained for the inhomogeneity in norm. It seems that the grains are separated into several groups for the magnitude of that inhomogeneity. 


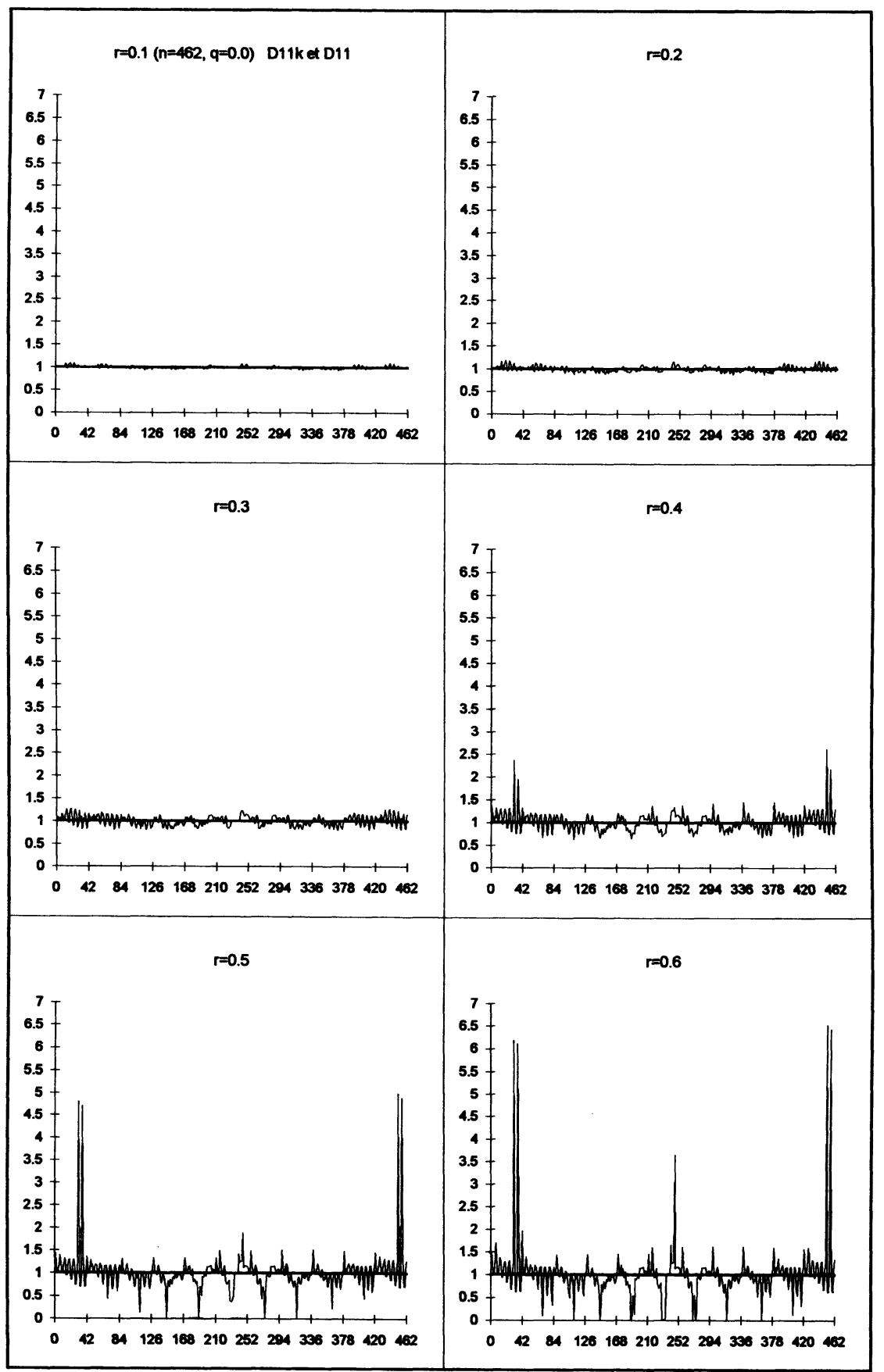

Figure 3 Value of the 1-1 component of strain-rate in the orientation number $k, D_{11}{ }^{k}$, as function of $k$, for different values of the strain-rate inhomogeneity $r$. The macroscopic value $D_{11}=1$ is reported. 


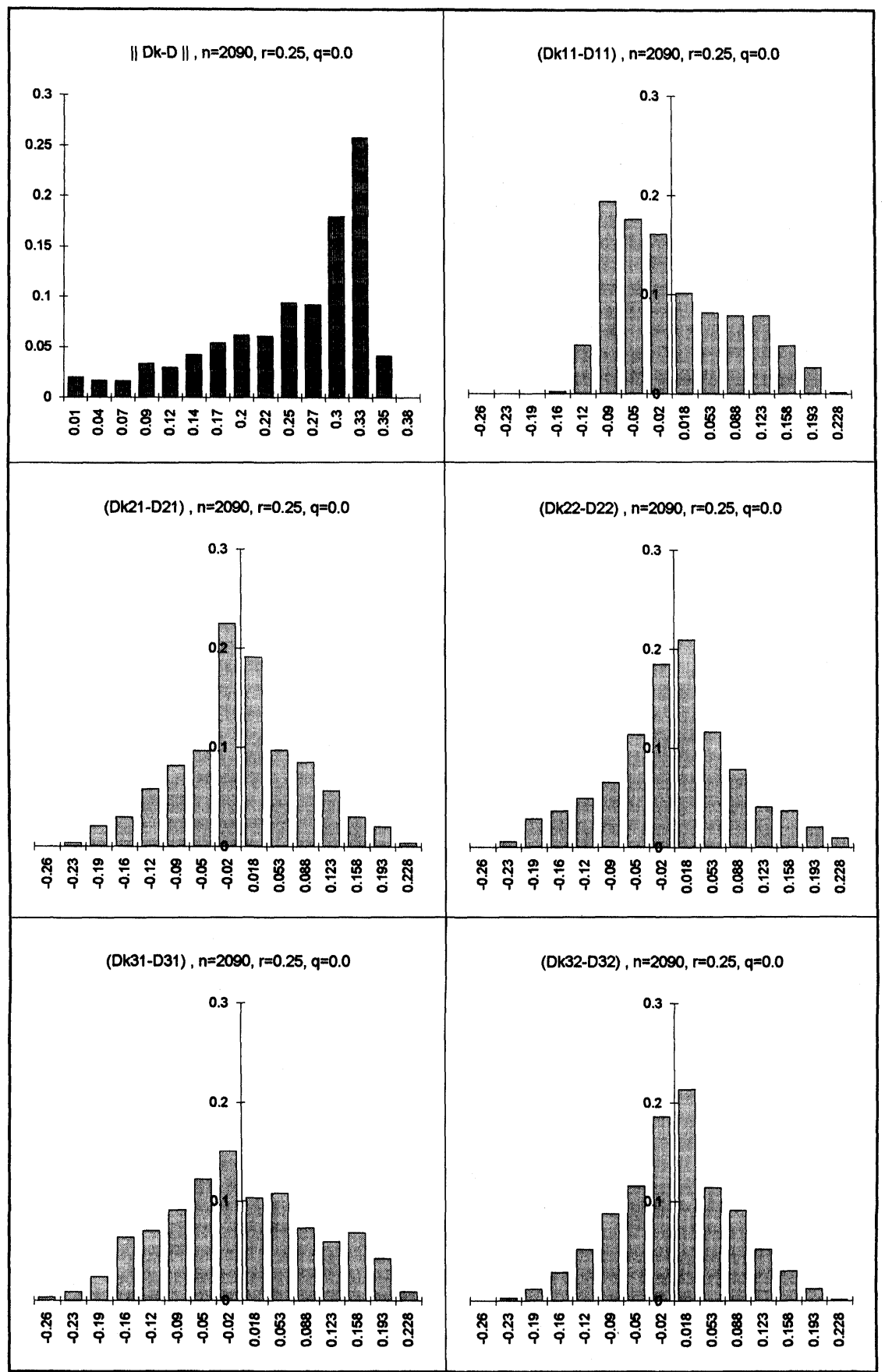

Figure 4 Histograms of the inhomogeneity in norm and the inhomogeneities in the independent components of strain-rate [cf. Eq. (27)], for $q=0$ (rolling) and for $r=0.25$. 


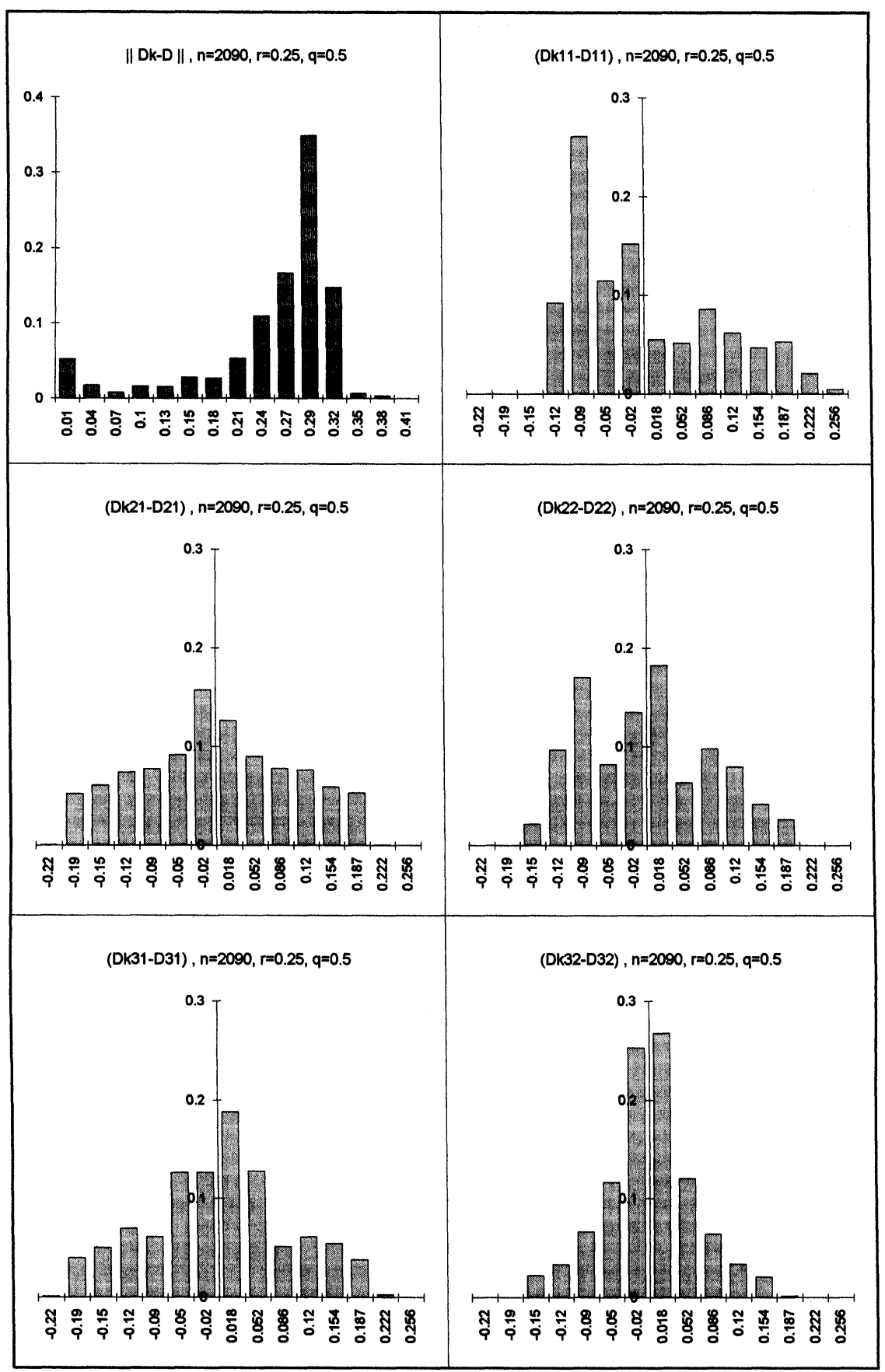

Figure 5 Histograms of the inhomogeneity in norm and the inhomogeneities in the independent components of strain-rate [cf. Eq. (27)], for $q=0.5$ (simple tension) and for $r=0.25$. 
Figure 6 shows the quadratic average inhomogeneities of the 6 different components, $a_{i j}=\left[\Sigma_{k} f_{k}\left(D^{k} i j-D_{i j}\right)^{2}\right]^{1 / 2}$ : these are not very different from one component to the other and depend also on the contraction coefficient $q$. In particular, for rolling ( $q=0$, thus IQ $=1)$, the most significant difference, according to the proposed model, is between the quadratic inhomogeneity of the $D_{13}$ component and those of the $D_{23}$ and $D_{12}$ components, the latter being respectively about $75 \%$ or $80 \%$ of the former. [Note that diagonal overall tensors $\mathbf{D}$ are considered here; hence, due to the constraint $\mathbf{D}^{*}=\mathbf{D}$ in the minimum problem (14), the average value of every shear component is exactly zero: see Eq. (19).] However, $D_{12}$ is assumed uniform in the relaxed Taylor model, thus zero inhomogeneity for $D_{12}$, whereas either $D_{13}$, or $D_{13}$ and $D_{23}$, are assumed completely "free" in the latter model, depending on the "lath" or "pancake" version (Van Houtte, 1981). In a finite element simulation of the rolling of an fcc polycrystal, Beaudoin et al. (1995) find a similar figure for the quadratic average inhomogeneities of the different shear components for equiaxed grains: they find indeed that the average inhomogeneity of both $D_{12}$ and $D_{23}$ is about $70 \%$ that of $D_{13}{ }^{4}$. That difference is found

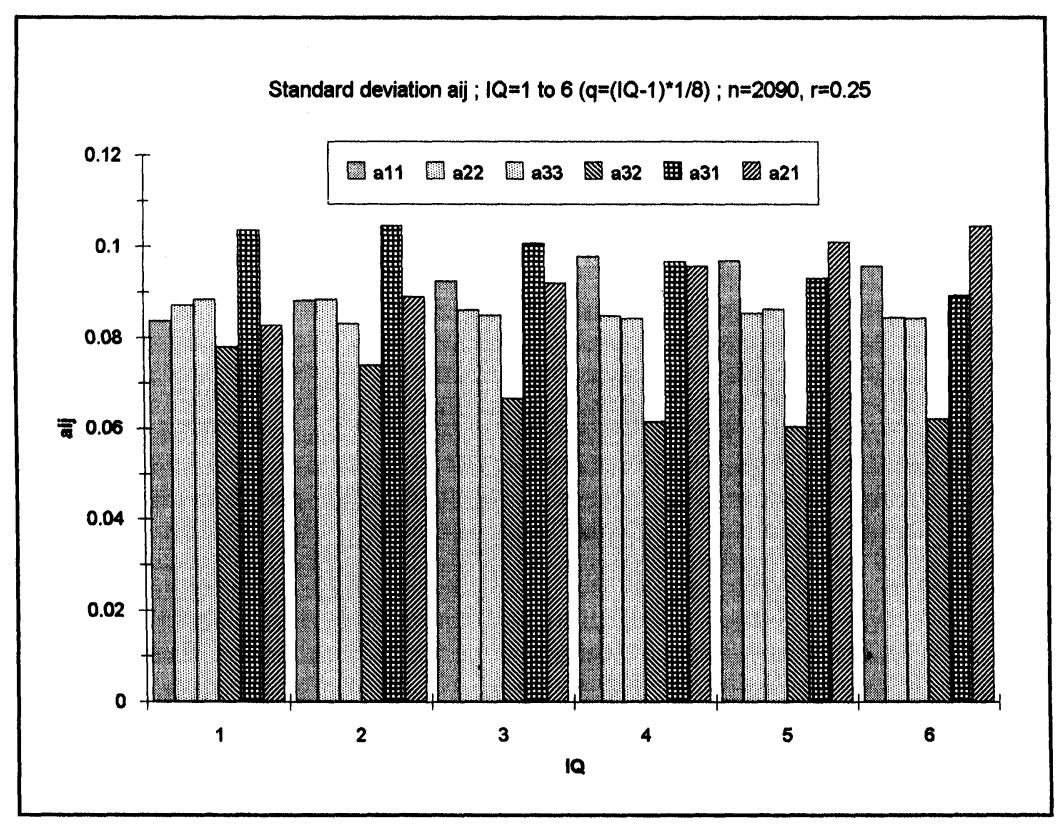

Figure 6 Standard deviations $a_{i j}$ of the different strain-rate components $D_{i j}$, as function of the contraction coefficient $q$ from 0 (rolling) to $q=5 / 8$ in steps of $1 / 8$, for $r=0.25$.

\footnotetext{
${ }^{4}$ They also find higher levels for the inhomogeneity of the shear components: about two times the levels we find for $r=0.25$. Although it is not indicated by Beaudoin et al., we expect that the inhomogeneity considered by them is that of the numerical field of strain-rate, thus between strainrates at different (Gauss) points, with several points inside each grain (finite element), or perhaps between strain-rates at different grains- in any case, not between strain-rates averaged over a crystal orientation as here. Thus they probably define the inhomogeneity at a smaller scale where it indeed should be larger than at the scale we define it. Anyhow, they consider an fcc material.
} 
by them to increase with the aspect ratio of the grains, in a way which is rather consistent with the relaxed Taylor model (although the average of the inhomogeneities of the different shear components is slightly decreased as the aspect ratio increases, which is clearly inconsistent with the relaxed Taylor model). However, it should be noted that, in their computations, "linear" 8-nodes elements have been chosen, thus rather rigid polyedric ones, and neighbouring finite elements have different orientations. These two facts tend to make their simulation closer to an ideal situation where the shape argument of the relaxed Taylor model could indeed apply, i.e. one with polyedric grains, each of which is rather homogeneously deformed. In particular, the result recalled in $\S 2.1$, which forbids a piecewise uniform strain field, does not apply to polyedric grains. According to the proposed model, there is also little difference between the inhomogeneities of the shear components and those of the diagonal ones, whereas the three diagonal components are assumed uniform in the relaxed Taylor model. In the model implemented for texture predictions for the work by Arminjon and Donadille (1990), the $D_{11}$ component was assumed uniform. Thus the present model indeed predicts a different inhomogeneity as compared with the "old" model.

\section{Deformation textures (steels): comparison with experiment and with Taylor's model}

A rather detailed investigation of experimental textures of several low-carbon steel sheets with weak or strong initial texture, deformed by cold-rolling, simple tension, plane strain tension, biaxial tension, and simple tension followed by biaxial tension, was carried out in previous works (e.g. Arminjon and Donadille, 1990). In those works, the experimental textures were systematically compared with textures predicted by the Taylor model and by a kind of "inhomogeneous Taylor model", hereafter named "old model", and different from the usual relaxed Taylor models. That "old model" consists in minimizing the power spent in each grain $(k)$ separately (among strain-rates $\mathbf{D}^{*^{k}}$ that belong to some prescribed neighborhood of D). It was considered by Arminjon and Donadille (1990) as an approximation of a more rigorous model, essentially the present model. ${ }^{5}$ Yet the present work represents, for polycrystals, the first implementation of the more rigorous model. The essential goal of the present computations was, therefore, to check whether the rigorous model confirms, and possibly improves, the previously observed agreement between experimental textures and textures predicted by using the old model. The ODF's predicted with the new model (be it with $r=$ 0.20 or with $r=0.25$ ) were indeed found to be very similar to those obtained with the old model, so the comparison will be based only on the "skeleton lines" $\alpha\left(\varphi_{1}\right.$ $\left.=0^{\circ}, \varphi_{2}=45^{\circ}\right), \varepsilon\left(\varphi_{1}=90^{\circ}, \varphi_{2}=45^{\circ}\right)$ and $\gamma\left(\Phi=55^{\circ}, \varphi_{2}=45^{\circ}\right)$.

\section{(i) Cold-rolling texture}

Figure 7 shows that $\alpha, \varepsilon$ and $\gamma$ lines for the cold-rolling texture at $75 \%$ reduction, either measured for a pure iron with very weak initial texture ("exp"), or calculated (using a $6^{\circ}$ Gaussian spread): with the Taylor model ("Tay"), or with the new model ( $r=0.20$ : "r020", $r=0.25$ : "r025"). The experimental agreement is far closer for the new model, either with $r=0.20$ or $r=0.25$, than for the Taylor model: this is apparent

\footnotetext{
${ }^{5}$ However, in Arminjon and Donadille (1990), the maximum inhomogeneity was considered instead of the average inhomogeneity. The present definition of the inhomogeneity is the relevant one for rate-independent rigid-plastic behaviour, because only $p=1$ meets the conditions used in the proofs given by Arminjon (1991a, Section 4).
} 


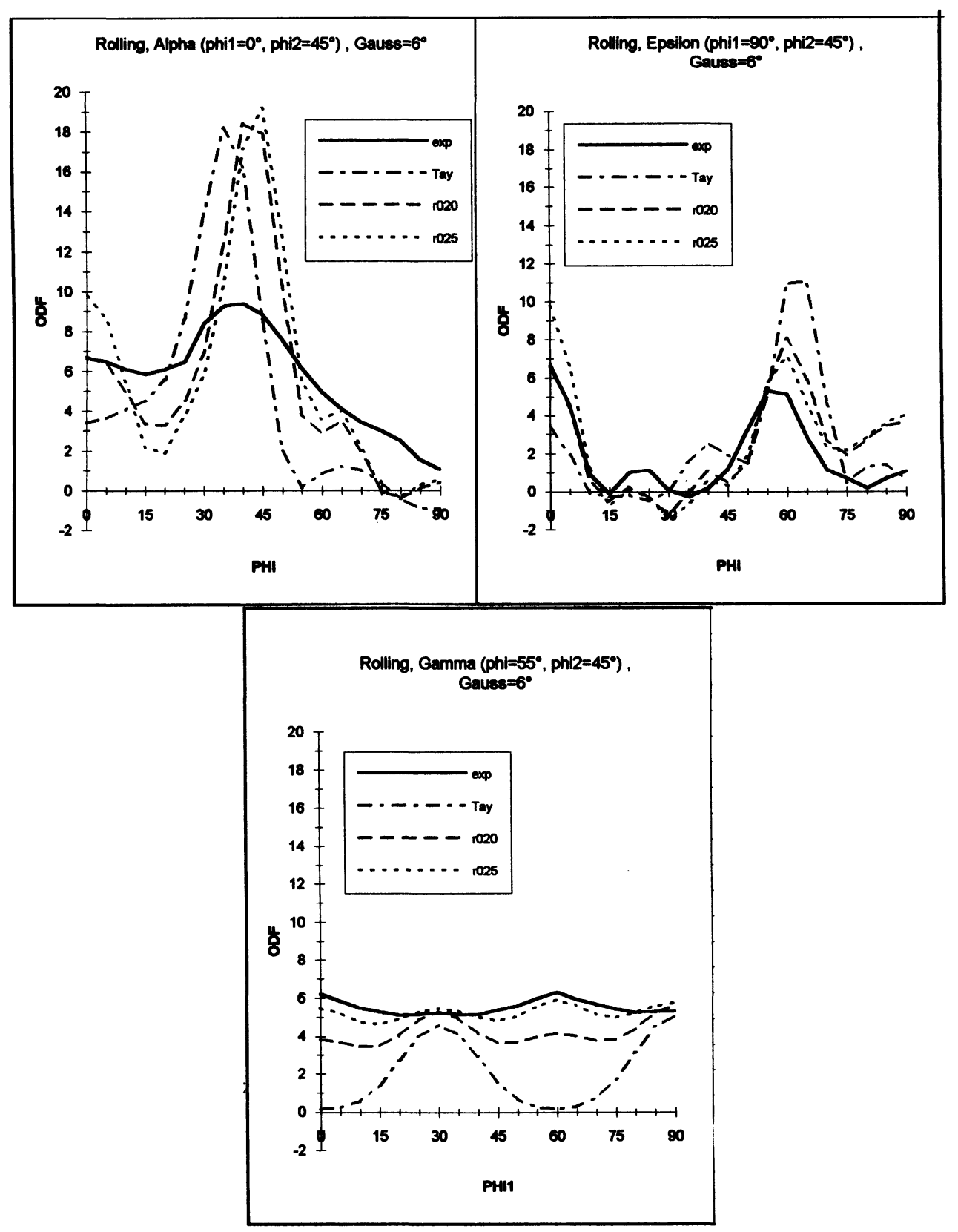

Figure 7 Skeleton lines of the $75 \%$ cold-rolling texture of a bcc metal, as measured for a pure iron (exp), or calculated either with the Taylor model (Tay) or with the proposed model $(r=0.20: r 020$, $r=0.25$ : r025). Gauss angle $\phi_{0}=6^{\circ}$. 
for all three fibers and, in particular, for the $\gamma$ fiber. However, the relative heights of the different peaks are better predicted with $r=0.25$ than with $r=0.20$, although all calculations overestimate the intensity on the main peak of the $\alpha$ line, the maximum of which is at $\Phi=35^{\circ}$ (Taylor), $40^{\circ}$ (measurement and $\left.r=0.20\right)$, or $45^{\circ}(r=0.25$ ). For the truncation order $l_{\max }=22$, a higher Gauss angle, $\phi_{0}>6^{\circ}$, is certainly more appropriate in terms of the "cleanness" of the obtained theoretical texture, although it artificially reduces its sharpness. In our opinion, any one-point model which does not include a random component, in the algorithm it uses to determine the strain distribution, must be expected to overestimate the sharpness of the texture. Taylor's model is only one well-known example, but the fact that it gives an upper bound to the yield surface does not imply that it must overestimate the sharpness of the texture more than other models. In order to compare two different models as to the sharpness of the predicted textures, it must be carefully checked whether the two numerical calculations are as accurate, for a less accurate calculation might give the impression of a better model in that respect! In the present case, the calculations for the Taylor model and the proposed model differ only at the stage of the determination of the strain distribution (which is trivial for the Taylor model), and the rather severe tolerances we have imposed for the proposed model at this stage (see above) give objective reasons to believe that both models are as accurately operated. So we acknowledge that the proposed model does not represent a decisive improvement as compared with the Taylor model, as to the sharpness of the predicted textures. ${ }^{6}$ Thus, to test more exactly the accuracy of the predicted textures, it is better to use a higher Gauss angle so that the experimental and theoretical ODF's have comparable sharpness (and again, $\phi_{0}=9^{\circ}$ is "cleaner" for $l_{\max }=22$ ). Figure 8 shows the same comparison as Figure 7, except that a $9^{\circ}$ Gaussian spread has been used, the "r020" calculation is not shown, and instead the prediction of the "old" model is shown. (Four lines seems to be a maximum to keep these figures readable; the effect of changing the Gauss angle is easy to guess.) With this Gauss angle, the difference between the experimental texture and the prediction of the new model (with $r=0.25$ ) is probably close to the experimental uncertainty. In particular, the new model does even better as the old one, due to the better predicted ratio between $\{001\}<110>\left(\Phi=0^{\circ}\right.$ on the $\alpha$ and $\varepsilon$ lines $)$ and the other peaks. We emphasize that, here, an important cause of experimental uncertainty, namely the effect of the initial texture, has been practically eliminated: the initial texture of this pure iron was very weak due to appropriate thermomechanical treatments (absolute maximum $\approx 2$ for the ODF), and a number of low-C steels with different compositions, all managed to have a very weak initial texture, lead to very similar cold-rolling textures (Arminjon and Donadille, 1990). (Moreover, in the latter work, this very weak initial texture was accounted for, which could not be done here, for the file was lost). This remark may be worth, because the question arises in the literature whether some rather important differences between experimental and predicted cold-rolling textures of iron or lowC steel could lie within the experimental band (Därmann et al., 1984). There is now no doubt that the Taylor model does not predict the cold-rolling texture of low-C steels within experimental band (Raphanel and Van Houtte, 1985; Arminjon and Donadille,

\footnotetext{
${ }^{6}$ However, to check this precisely, it would be necessary to refine the grid used in the interpolation procedure for the rotation field, because the $10^{\circ}$ step probably contributes to make the textures less sharp. This may partly mask the difference in the sharpness of the textures which should be predicted by both models if exactly run.
} 


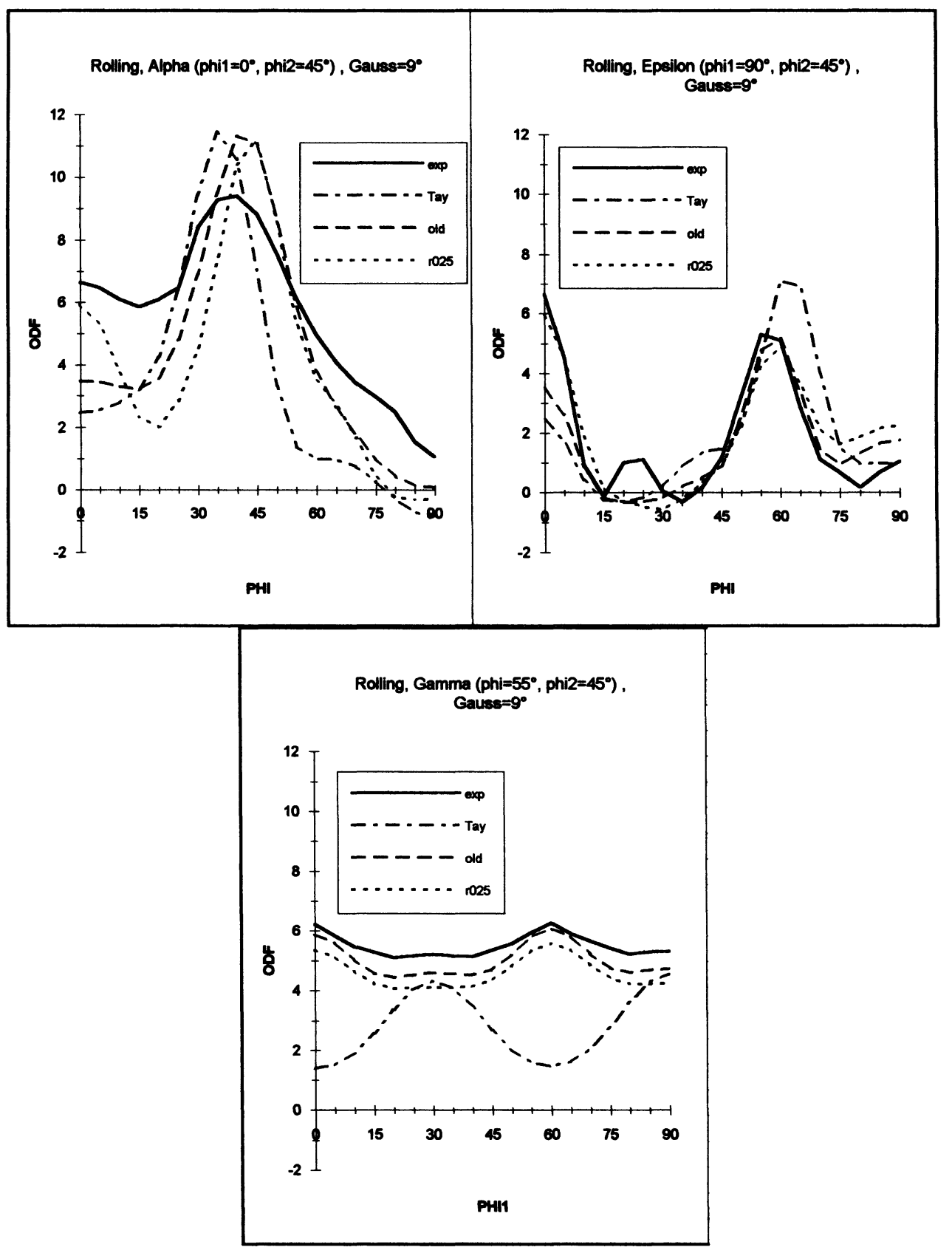

Figure 8 Skeleton lines of the $75 \%$ cold-rolling texture of a bcc metal, as measured for a pure iron (exp), or calculated either with the Taylor model (Tay), with the former model (old), or with the proposed model $(r=0.25$ : $r 025)$. Gauss angle $\phi_{0}=9^{\circ}$. 
1990; Wagner et al., 1991). It is also clear that the "old" model is much closer from doing this, and that the new model is still closer to experiment than the old one. Moreover, it is apparent from a comparison of this work with the work by Wagner et al. (1991) that the proposed model predicts cold-rolling textures that are closer to the experimental ones than does the relaxed Taylor model or even the viscoplastic selfconsistent code used by Wagner et al. Of course, an entirely convincing proof of the latter statement would demand to make a comparison for the same material.

(ii) Simple and biaxial tension

Figure 9 shows the comparison between the skeleton lines of the experimental and theoretical ODF's of an Al-killed steel [denoted 1], as the same steel was denoted in Arminjon and Donadille (1990) and in Arminjon and Imbault (1994)], deformed by $\varepsilon=0.30$ in simple tension in the transverse direction. A Gauss angle $\phi_{0}=6^{\circ}$ has been taken for the theoretical textures, which turns out to give the same levels for the experimental and theoretical ODF's (it is not surprising that a stronger overestimate of the sharpness of the texture was found at the much higher strain undergone in rolling, $\varepsilon=1.39$ ). Although the difference between the two models is smaller than for coldrolling (which is expected, for the same reason), it remains true that the new model (with $r=0.25$ as for cold-rolling), gives a better agreement with experiment than does the Taylor model. This may be seen also for the theoretical values of the Lankford coefficient, calculated from the experimental or theoretical deformation textures using the texture-adjusted fourth-order dual potential (see e.g. Arminjon and Imbault, 1994, and references therein, for details about this dual potential and its ability to predict correct values of the Lankford coefficient from the as-received texture). The $R$ values after prestraining may be influenced by not only the texture evolution, but their calculation from the deformation textures is a way to translate the differences in a mechanically more relevant way. It is also seen that the new model does a slightly better job than does the old one.

Finally Figure 10 shows just the same comparison as Figure 9, but this time for the textures after $\varepsilon \equiv \varepsilon_{1}+\varepsilon_{2}=0.52$ in biaxial tension, and just the same conclusions may be drawn as to the relative performance of the Taylor model, the "old" model, and the new model.

\section{CONCLUSIONS}

1. Volume-fraction models or "one-point" models are the easiest to use among micromacro models, in particular they are for the moment the only ones that lead to tractable computations for deformation textures. But to justify the use of such models, a number of statistical assumptions, stated here in a simple form, have to be set. The "grains" considered by such models must be interpreted as zones with given state, e.g. zones with given crystal orientation, which in general involve many separated grains. The stresses and strains in the "grains", predicted by such models, must be interpreted as approximations of the average values of the actual fields of stress and strain for a given orientation.

2. If, to these assumptions of statistical homogeneity, one merely adds the maximum work principle, then one obtains a tractable form of the lower bound and the upper bound theorems, the upper bound corresponding to the Taylor model. That form of bounds is tractable in that it is expressed in terms of the discrete distribution 


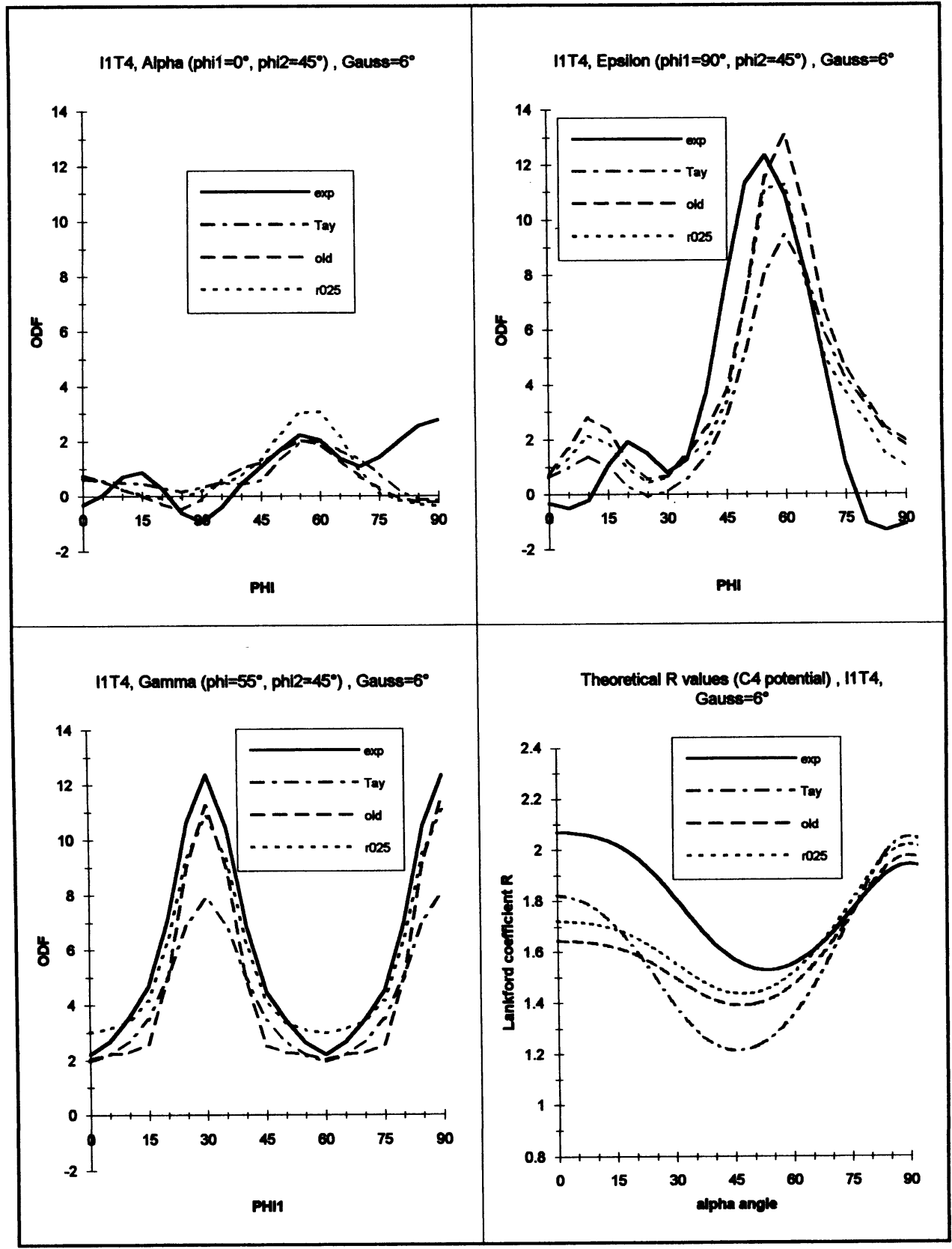

Figure 9 Skeleton lines of the texture of the Al-killed steel I1 after 0.30 strain in simple tension, as measured (exp), or calculated either with the Taylor model (Tay), with the former model (old), or with the proposed model $(r=0.25$ : $r 025)$. Gauss angle $\phi_{0}=6^{\circ}$. Also shown are the theoretical values of the Lankford coefficient $R(\alpha)$ ( $\alpha$ from $0^{\circ}$ to $90^{\circ}$ ), calculated from those experimental and theoretical textures by using the $4^{\text {th }}$ order dual (C4) potential. 


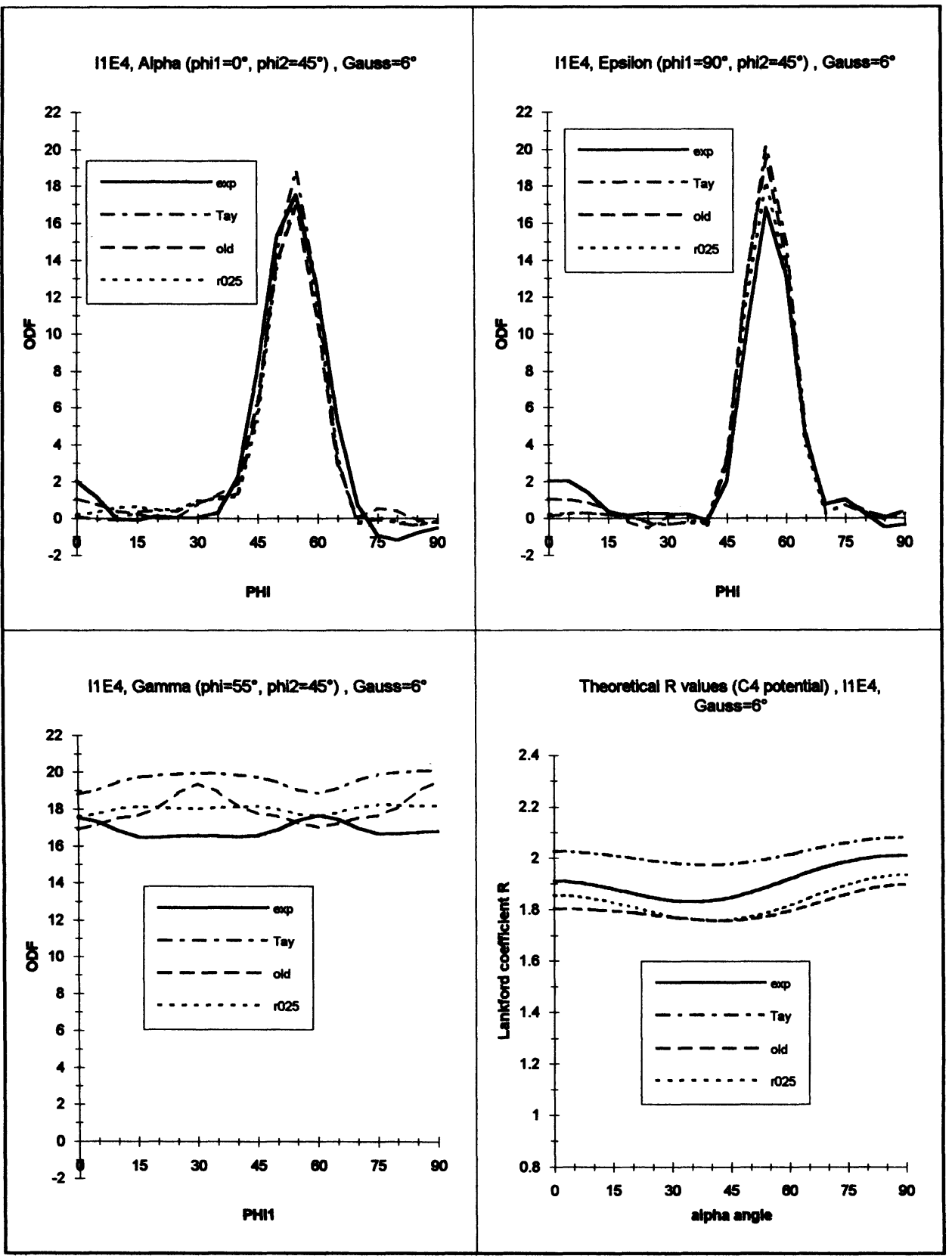

Figure 10 Same as Figure 9, but here after 0.52 strain in biaxial tension. 
of the average strains in the "grains" (orientations), instead of the continuous fields. We introduce the average inhomogeneity $h$ of any possible distribution of that average strains. Under the same assumptions, we have the following result: there exists a value $r_{0}$ of $h$, such that the actual power spent at the macroscopic scale is the minimum of the average power spent in the "grains" among those distributions of strain that have this inhomogeneity $r_{0}$. We assume that the strain distribution giving the minimum is the actual distribution of the strains among the different "grains".

3. This model has been numerically implemented for polycrystals and applied to the prediction of strain distribution and deformation textures. The histograms of the strain (-rates) have a nice appearance, in several cases they look like histograms of Gaussian distributions. However, the calculation of the strain distribution is entirely deterministic. Only small differences between the average inhomogeneity of the different strain components (e.g. between different shear components, or between shear and diagonal components) are predicted by the proposed model.

4. The cold-rolling textures of low-C steels are far better predicted by this model than by the Taylor model. Except for the sharpness of the texture, which remains overestimated, the predicted texture coincides with the measured texture nearly within the experimental accuracy. Thus this prediction seems also more accurate than those obtained by using the relaxed Taylor model or the viscoplastic self-consistent code, although the calculations of the latter models do not correspond to the same material as here. For simple and biaxial tension (imposed to a textured steel), there is less difference between different models due to the smaller strains involved, but still the proposed model does better than does the Taylor model. Finally, the present rigorous model predicts deformation textures that are close to those obtained by the more empirical model used previously. This justifies a posteriori the use of the older model as an approximation of the present one.

\section{References}

Arminjon, M. (1991a). Limit distributions of the states and homogenization in random media. Acta Mech. 88, 27-59.

Arminjon, M. (1991b). Macro-homogeneous strain fields with arbitrary local inhomogeneity. Arch. Mech. 43, 191-214.

Arminjon, M., Chambard, T. and Turgeman, S. (1994). Variational micro-macro transition, with application to reinforced mortars. Int. J. Solids Structures 31, 683-704.

Arminjon, M. and Donadille, C. (1990). Présentation d'un modèle polycristallin extrémal. Application aux aciers d'un modèle approché. Mém. Et. Sci. Revue Métall. 87, 359-382.

Arminjon, M. and Imbault, D. (1993). Does a polycrystal model disclose a single plastic spin? In Large Plastic Deformations. C. Teodosiu, J.L. Raphanel and F. Sidoroff, eds., Balkema, Rotterdam, pp. 89-99.

Arminjon, M. and Imbault, D. (1994). An analytical micro-macro model for textured polycrystals at large plastic strains. Int. J. Plasticity, 10, 825-847.

Beaudoin, A.J., Dawson, P.R., Mathur, K.K. and Kocks, U.F. (1995). A hybrid finite element formulation for polycrystal plasticity with consideration of macrostructural and microstructural linking. Int. J. Plasticity, 11, 501-521.

Berveiller, M. and Zaoui, A. (1979). An extension of the self-consistent scheme to plastically flowing polycrystals. J. Mech. Phys. Solids, 26, 325-344.

Bishop, J.F.W. and Hill, R. (1951). A theory of the plastic distortion of a polycrystalline aggregate under combined stresses. Phil. Mag. 42, 414-427.

Bunge, H.J. (1969). Mathematische Methoden der Texturanalyse. Akademie Verlag, Berlin.

Därmann, C., Mishra, S. and Lücke, K. (1984). On the rolling texture in bcc materials and comparison to theory. In Proc. ICOTOM 7. C.M. Brakman et al., eds., Netherl. Soc. Mater. Sci., Zwijndrecht, pp. $47-51$.

Hill, R. (1950). The Mathematical Theory of Plasticity. Clarendon Press, Oxford. 
Hill, R. (1952). The elastic behaviour of a crystalline aggregate Proc. Phys. Soc. Lond. A65, 349-354.

Hill, R. (1967). The essential structure of constitutive laws for metal composites and polycrystals. J. Mech. Phys. Solids, 15, 79-95.

Kern, R. and Bunge H.J. (1984). Texture and microstructure of cold-rolled high-purity iron. In Proc. ICOTOM 7. C.M. Brakman et al., eds., Netherl. Soc. Mater. Sci., Zwijndrecht, pp. 89-94.

Kocks, U.F. and Canova, G.R. (1981). How many slip systems, and which? In Deformation of Polycrystals (Proc. Risø Conf.) N. Hansen et al., eds., Risø National Laboratory, Roskilde (Dk.), pp. $35-44$.

Kocks, U.F. and Chandra, H. (1982). Slip geometry in partially constrained deformation. Acta Metall. 30, 695-709.

Leffers, T. (1968). Computer simulation of the plastic deformation in face-centred cubic polycrystals and the rolling texture derived. Ris $\varnothing$ report No. 184.

Leffers, T. and Juul Jensen, D. (1991). The relation between texture and microstructure in rolled fcc materials. Textures and Microstructures 14-18, 933-952.

Leffers, T., Sørensen, N.J. and Lebensohn, R.A. (1995). The uncertainty in the calculation of the changes in crystallographic orientation which lead to deformation texture. In Dynamic Plasticity and Structural Behaviors (Proc. Plasticity '95). Sh. Tanimura and A.S. Khan, eds., Gordon \& Breach, Luxembourg, pp. 273-276.

Mecking, H. (1980). Deformation of polycrystals. In Strength of Metals and Alloys (Proc. ICSMA 5). P. Haasen et al., eds., Pergamon, Oxford, pp. 1573-1594.

Pedersen, O.B. and Leffers, T. (1987). Modelling of plastic heterogeneity in deformation of singlephase materials. In Constitutive Relations and their Physical Basis (Proc. Ris $\emptyset$ Conf.). S.I. Andersen et al., eds. Risø National Laboratory, Roskilde (Dk.), pp. 147-172.

Raphanel, J.L. and Van Houtte, P. (1985). Simulation of the rolling textures of bcc metals by means of the relaxed Taylor theory. Acta Metall. 33, 1481-1488.

Renouard, M. and Wintenberger, M. (1976). Déformation homogène par glissements de dislocations de monocristaux de structure cubique faces centrées sous l'effet de contraintes et de déplacements imposés. C. R. Acad. Sci. Paris, 283 B, 237-240.

Royer, F., Tavard, C. and Penning, P. (1979). Recherche des systèmes de glissement actifs dans un processus de deformation plastique du polycristal. II. Cas du glissement non-cristallographique dans les métaux cubiques. J. Appl. Cryst. 12, 436-441.

Sachs, G. (1928). Zur Ableitung einer Fließbedingung. Z. Vereins Deutsch. Ing. 72, 734 ff.

Taylor, G.I. (1938). Plastic strain in metals. J. Inst. Metals 62, 307-324.

Van Houtte, P. (1981). Adaptation of the Taylor theory to the typical substructure of some cold-rolled f.c.c. materials. In Proc. ICOTOM 6. S. Nagashima et al., eds., Iron \& Steel Inst. Japan, Tokyo, pp. $428-437$.

Van Houtte, P. (1982). On the equivalence of the relaxed Taylor theory and the Bishop-Hill theory for partially constrained plastic deformation of crystals. Mater. Sci. Eng. 55, 69-77.

Van Houtte, P. (1995). Heterogeneity of plastic strain around an ellipsoidal inclusion in an ideal plastic matrix. Acta Metall. Mater. 43, 2859-2879.

Voigt, W. (1887). Theoretische Studien über die Elastizitätsverhältnisse der Kristalle. Abh. Kgl. Ges. Wiss. Göttingen, math. Kl. 34, $47 \mathrm{ff}$.

Wagner, F., Canova, G., Van Houtte, P. and Molinari, A. (1991). Comparison of experimental and simulated deformation textures for bcc metals. Textures and Microstructures 14-18, 1135-1140. 